\title{
Classification and Asymptotic Scaling of Hadrons' Light-Cone Wave-Function Amplitudes
}

\author{
Xiangdong Ji,, , Jian-Ping Ma,, 丹 and Feng Yuan ${ }^{1, f}$ \\ ${ }^{1}$ Department of Physics, University of Maryland, \\ College Park, Maryland 20742, USA \\ ${ }^{2}$ Institute of Theoretical Physics, Academia Sinica, Beijing, 100080, P. R. China
}

(Dated: October 30, 2018)

\begin{abstract}
We classify the hadron light-cone wave-function amplitudes in terms of parton helicity, orbital angular momentum, and quark-flavor and color symmetries. We show in detail how this is done for the pion, $\rho$ meson, nucleon, and delta resonance up to and including three partons. For the pion and nucleon, we also consider four-parton amplitudes. Using the scaling law derived previously, we show how these amplitudes scale in the limit that all parton transverse momenta become large.
\end{abstract}

*Electronic address: xji@physics.umd.edu

${ }^{\dagger}$ Electronic address: majp@itp.ac.cn

${ }^{\ddagger}$ Electronic address: fyuan@physics.umd.edu 


\section{INTRODUCTION}

Although the hadron structure is believed to be described by the fundamental theory of strong interactions - quantum chromodynamics (QCD), the actual solution of the problem is notoriously difficult to achieve. Apart from numerically solving QCD on a spacetime lattice, there is no other systematic theoretical approach that has been very successful. The closest might be the light-front quantization approach in which the old-fashioned method of diagonalizing a hamiltonian is followed [1, 2]. The conceptual advantage here is obvious: Hadrons are described by light-cone wave functions which have clear physical meaning and are very useful phenomenologically, whereas in lattice QCD the natural language is classical gluon configurations, such as instantons and monopoles, in the Euclidean space. As to why light-cone quantization is superior compared to equal-time quantization, we just wish to point out that the vacuum structure in the former approach, which consists of just $k^{+}=0$ particles, can be easily separated from the part of the hadron structure consisting $k^{+} \neq 0$ particles. Moreover, in high-energy scattering hadrons travel at the near speed of light, and the light-cone coordinates appear naturally.

To be sure, there are many difficulties that one must clear before a realistic light-cone description of hadrons becomes possible. One of them is that hadrons are now described by an infinite number of light-cone Fock amplitudes, and there is no apparent reason why the amplitudes with 100 partons (quarks and gluons) are strongly suppressed relative to those with 2 or 3 partons. The answer, of course, depends on the choice of gauge, ultraviolet and infrared cut-offs, and ultimately the underlying QCD dynamics. One way to check is to truncate the Hilbert space first to including the partons to a maximum number $n$ and then to determine how the solution changes when the Fock components with $n+1$ number of partons are included. The optimistic view has been that since the constituent quark models work so well phenomenologically, there must exist a light-cone description of hadrons in which only the Fock components with a few partons are necessary. In high-energy exclusive processes, we know for sure that only the wave-function components with a few partons are relevant.

In the light-front description of a hadron, the very first step is to classify independent wave-function amplitudes given a particular parton combination. To our knowledge, there has not been much systematic study in the literature along this direction. In Ref. [3], we have proposed an approach by writing down the matrix elements of a class of light-cone-correlated quark-gluon operators, in much the same way that has been used to construct independent light-cone distribution amplitudes in which the parton transverse momenta are integrated out [4]. In Ref. [5], we have applied the approach to the nucleon, finding that six amplitudes are needed to describe the three-quark sector of the nucleon wave function. However, using the approach to handle Fock states with more partons appears to be complicated.

In [6], we have developed a more direct method to write down the general structure of the light-cone wave function for $n$ partons with orbital angular momentum projection $l_{z}$. We have also found a general power counting rule which determines the asymptotic behavior of the light-cone amplitudes when the transverse momenta of all partons become large. From the wave-function counting rule, we have derived the dimensional scaling law for high-energy exclusive processes including parton orbital angular momentum [7, 8]. In this paper, we report further progress in this direction. In particular, we use the method to classify the higher Fock components of hadrons. We will consider in detail how the flavor (for quarks) and color degrees of freedom of the partons are systematically coupled. We 
will write down the amplitudes for the pion and proton up to four partons. We also work out the leading light-cone wave functions for the $\Delta$ and $\rho$ meson, leaving more complicated cases for the readers.

Based on our work here, one can go on to parameterize the light-cone wave function amplitudes and fit to the experimental data. Although the amplitudes thus determined are phenomenological, they can be made to obey the asymptotic behavior at large transverse momenta 6]. Therefore, our result can provide guidelines for phenomenological studies for exclusive processes 9, 10, 11, 12. By committing ourselves to the light-cone amplitudes, we are also committing to the light-cone gauges $A^{+}=0$ 13, 14]. One subtlety about the lightcone gauge is that it requires additional gauge fixing [15, 16, 17]. Depending on whether the additional gauge condition is time-reversal invariant or not, the wave function amplitudes are real or fully complex. In the latter case, the final state interaction effects might be included in the amplitudes $[18,19,20,21]$. A related issue is that the light-cone amplitudes have light-cone singularities at small $x$ which require regularization.

Our plan of the presentation is as follows. We start in Sec. II by describing a general strategy to classify the independent wave-function amplitudes for a hadron state with a specific parton content. In Sec. III, we apply this method to write down the amplitudes of $\pi^{+}$for up to four-parton Fock components. We extend these discussions to $\rho$ mesons in Sec. IV, where the amplitudes up to three-parton Fock components will be given. In Sec. V, the proton wave-function amplitudes for three-quark and three-quark plus one-gluon Fock components will be presented. The leading results for the delta resonance will be given in Sec. VI. The final section contain a brief summary and outlook.

\section{GENERAL STRATEGY AND SYMMETRY CONSTRAINTS}

In this section, we discuss our general strategy in classifying and enumerating the hadron light-cone amplitudes. The goal is to find a simple and general way to write done all possible light-cone amplitudes of a hadron once a parton content is specified. In Sec. II.A, we explain our notation and convections. In Sec. II.B, we consider the helicity and angular momentum structure of a general Fock component. In Sec. II.C we make general comments about flavor and color structure. In Sec. II.D and E, we consider the parity and time reverse constraints on the light-cone wave function amplitudes.

\section{A. Notation}

We work in the framework of light-cone (or light-front) quantization 1, 14]. The lightcone time $x^{+}$and coordinate $x^{-}$are defined as $x^{ \pm}=1 / \sqrt{2}\left(x^{0} \pm x^{3}\right)$. Likewise we define Dirac matrices $\gamma^{ \pm}=1 / \sqrt{2}\left(\gamma^{0} \pm \gamma^{3}\right)$. The projection operators for Dirac fields are defined as $P_{ \pm}=(1 / 2) \gamma^{\mp} \gamma^{ \pm}$. Any Dirac field $\psi$ can be decomposed into $\psi=\psi_{+}+\psi_{-}$with $\psi_{ \pm}=P_{ \pm} \psi$. $\psi_{+}$is a dynamical degrees of freedom and has the canonical expansion,

$$
\begin{aligned}
\psi_{+}\left(\xi^{+}=0, \xi^{-}, \xi_{\perp}\right)= & \int \frac{d^{2} k_{\perp}}{(2 \pi)^{3}} \frac{d k^{+}}{2 k^{+}} \sum_{\lambda}\left[b_{\lambda}(k) u(k \lambda) e^{-i\left(k^{+} \xi^{-}-\vec{k}_{\perp} \cdot \vec{\xi}_{\perp}\right)}\right. \\
& \left.+d_{\lambda}^{\dagger}(k) v(k \lambda) e^{i\left(k^{+} \xi^{-}-\vec{k}_{\perp} \cdot \vec{\xi}_{\perp}\right)}\right]
\end{aligned}
$$


where $b^{\dagger}(b)$ and $d^{\dagger}(d)$ are quark and antiquark creation (annihilation) operators, respectively. We adopt covariant normalization for the particle states and the creation and annihilation operators, i.e.,

$$
\left\{b_{\lambda}(k), b_{\lambda^{\prime}}^{\dagger}\left(k^{\prime}\right)\right\}=\left\{d_{\lambda}(k), d_{\lambda^{\prime}}^{\dagger}\left(k^{\prime}\right)\right\}=(2 \pi)^{3} \delta_{\lambda \lambda^{\prime}} 2 k^{+} \delta\left(k^{+}-k^{\prime}\right) \delta^{(2)}\left(\vec{k}_{\perp}-\vec{k}_{\perp}^{\prime}\right),
$$

where $\lambda$ is the light-cone helicity of the quarks which can take $+1 / 2$ or $-1 / 2$. We ignore the masses of the light up and down quarks. Later, to simply the notations, we simply use $u^{\dagger}$ and $\bar{u}^{\dagger}$ to represent creation operators for up and anti-up quarks, respectively, and so on.

Likewise, for the gluon fields in the light-cone gauge $A^{+}=0, A_{\perp}$ is dynamical and has the expansion,

$$
\begin{aligned}
A_{\perp}\left(\xi^{+}=0, \xi^{-}, \xi_{\perp}\right)= & \int \frac{d^{2} k_{\perp}}{(2 \pi)^{3}} \frac{d k^{+}}{2 k^{+}} \sum_{\lambda}\left[a_{\lambda}(k) \epsilon(k \lambda) e^{-i\left(k^{+} \xi^{-}-\vec{k}_{\perp} \cdot \vec{\xi}_{\perp}\right)}\right. \\
& \left.+a_{\lambda}^{\dagger}(k) \epsilon^{*}(k \lambda) e^{i\left(k^{+} \xi^{-}-\vec{k}_{\perp} \cdot \vec{\xi}_{\perp}\right)}\right] .
\end{aligned}
$$

Implicitly, the gauge fields $A^{\mu}$ is a traceless $3 \times 3$ matrix with $A^{\mu}=\sum_{a} A^{a \mu} T^{a}$, where $T^{a}$ are the $S U(3)$ Gell-Mann matrices satisfying $\left[T^{a}, T^{b}\right]=i f^{a b c} T^{c}$ and $\left\{T^{a}, T^{b}\right\}=\frac{1}{3} \delta_{a b}+d_{a b c} T^{c}$. Again, we have the following covariant normalization for the creation and annihilation operators for gluon,

$$
\left[a_{\lambda}(k), a_{\lambda^{\prime}}^{\dagger}\left(k^{\prime}\right)\right]=(2 \pi)^{3} \delta_{\lambda \lambda^{\prime}} 2 k^{+} \delta\left(k^{+}-k^{\prime}\right) \delta^{(2)}\left(\vec{k}_{\perp}-\vec{k}_{\perp}^{\prime}\right)
$$

Later, we simply use $g^{\dagger}$ to represent a gluon creation operator. $\psi_{-}$and $A^{-}$are dependent variables, which can be expressed in terms of $\psi_{+}$and $A_{\perp}$ using equations of motion [13].

\section{B. Angular Momentum Structure}

For a given parton content, i.e., a specification of quarks, antiquarks and gluons, the light-cone amplitudes of a hadron with helicity $\Lambda$ can be classified in terms of the total parton light-cone helicity $\lambda$. The angular momentum conservation then demands that the partons have angular momentum projection $l_{z}=\Lambda-\lambda$. Let us find the angular momentum structure of the amplitudes satisfying these conditions [6].

Suppose a Fock component has $n$ partons with creation operators $a_{1}^{\dagger}, \ldots, a_{n}^{\dagger}$, where the partons can either be gluons or quarks and the subscripts label the partons' quantum numbers such as spin, flavor, color, momentum, etc. Assume all color, flavor (for quarks) indices have been coupled properly using Clebsch-Gordon coefficients (see next subsection). The longitudinal momentum fractions of the partons are $x_{i}(i=1,2, \ldots, n)$, satisfying $\sum_{i=1}^{n} x_{i}=1$, and

the transverse momenta $\vec{k}_{1 \perp}, \ldots, \vec{k}_{n \perp}$, satisfying $\sum_{i}^{n} \vec{k}_{i \perp}=0$. We will eliminate $\vec{k}_{n \perp}$ in favor of the first $n-1$ transverse momenta. Assume the orbital angular momentum projections of the partons are $l_{z 1}, \ldots, l_{z(n-1)}$, respectively, and let $l_{z}=\sum_{i=1}^{n-1} l_{z i}$, then

$$
l_{z}+\lambda=\Lambda,
$$

where $\lambda=\sum_{i=1}^{n} \lambda_{i}$ is the total parton helicity. Without loss of generality, we assume $l_{z} \geq 0$; even then, $l_{z i}$ can have both signs. Thus, a general term in the hadron wave function 
amplitude has the structure

$$
\int \prod_{i=1}^{n} d[i]\left(k_{1 \perp}^{ \pm}\right)^{\left|l_{z 1}\right|}\left(k_{2 \perp}^{ \pm}\right)^{\left|l_{z 2}\right|} \ldots\left(k_{(n-1) \perp}^{ \pm}\right)^{\left|l_{z(n-1)}\right|} \psi_{n}\left(x_{i}, k_{i \perp}, \lambda_{i}, l_{z i}\right) a_{1}^{\dagger} a_{2}^{\dagger} \ldots a_{n}^{\dagger}|0\rangle,
$$

where $k_{i \perp}^{ \pm}=k_{i}^{x} \pm k_{i}^{y}$ and the $+(-)$ sign applies when $l_{z i}$ is positive (negative), and

$$
d[i]=d x_{i} d^{2} k_{i \perp} /\left(\sqrt{2 x_{i}}(2 \pi)^{3}\right)
$$

with the overall constraint on $x_{i}$ and $k_{i \perp}$ implicit.

The above form can be further simplified as follows. Assume $l_{z i}$ is positive and $l_{z j}$ negative, and $l_{z i}>\left|l_{z j}\right|$, we have

$$
\begin{aligned}
\left(k_{i \perp}^{+}\right)^{l_{z i}}\left(k_{j \perp}^{-}\right)^{-l_{z j}} & =\left(k_{i \perp}^{+}\right)^{l_{z i}+l_{z j}}\left(k_{i \perp}^{+} k_{j \perp}^{-}\right)^{-l_{z j}} \\
& =\left(k_{i}^{+}\right)^{l_{z i}+l_{z j}}\left(\vec{k}_{i \perp} \cdot \vec{k}_{j \perp}-i \epsilon^{\alpha \beta} k_{i \alpha} k_{j \beta}\right)^{-l_{z j}} \\
& =\left(k_{i \perp}^{+}\right)^{l_{z i}+l_{z j}}\left(\phi_{0}+\phi_{1} i \epsilon^{\alpha \beta} k_{i \alpha} k_{j \beta}\right),
\end{aligned}
$$

where $\alpha, \beta=1,2, \phi_{0,1}$ are polynomials in $\vec{k}_{i \perp}^{2}, \vec{k}_{j \perp}^{2}$, and $\vec{k}_{i \perp} \cdot \vec{k}_{j \perp}$. On the last line of the above equation we have used the identity $\epsilon^{\alpha \beta} \epsilon^{\gamma \delta}=\delta^{\alpha \gamma} \delta^{\beta \delta}-\delta^{\alpha \delta} \delta^{\beta \gamma}$. If $l_{z i}+l_{z j} \neq 0$, one can

use $i \epsilon^{\alpha \beta} k_{1 \alpha} k_{2 \beta} k_{1 \perp}^{+}=\vec{k}_{1 \perp} \cdot \vec{k}_{2 \perp} k_{1 \perp}^{+}-\vec{k}_{1 \perp} \cdot \vec{k}_{1 \perp} k_{2 \perp}^{+}$to further reduce the second term in the bracket. Following the above procedure, we can eliminate all negative $l_{z j}$, a general $l_{z}>0$ component in the wave function reads

$$
\begin{aligned}
& \int \prod_{i=1}^{n} d[i]\left(k_{1 \perp}^{+}\right)^{l_{z 1}}\left(k_{2 \perp}^{+}\right)^{l_{z 2}} \ldots\left(k_{(n-1) \perp}^{+}\right)^{l_{z(n-1)}} \\
& \quad \times\left(\psi_{n}\left(x_{i}, k_{i}, \lambda_{i}, l_{z i}\right)+\sum_{\left.i<j=\left.1\right|_{l_{z i}=l_{z j}=0} ^{n-1} i \epsilon^{\alpha \beta} k_{i \alpha} k_{j \beta} \psi_{n(i j)}\left(x_{i}, k_{i \perp}, \lambda_{i}, l_{z i}\right)\right) a_{1}^{\dagger} a_{2}^{\dagger} \ldots a_{n}^{\dagger}|0\rangle}\right.
\end{aligned}
$$

where $\sum_{i} l_{z i}=l_{z}$ and $l_{z i} \geq 0$, and the sums over $i$ and $j$ are restricted to the $l_{z i}=0$ partons. The above equation is our starting point to write down independent light-cone amplitudes.

\section{Flavor and Color Structure}

For a given quark content, we classify the amplitudes in terms of the flavor symmetry. For instance, for the pion state, we need to project out the Fock component with the total isospin 1. The problem becomes more involved if a Fock state contains many quark-antiquark pairs because there are more than one way to construct the states with the definite isospin.

Our general strategy is as follows: we first consider all possible ways to construct the same isospin. We then use the freedom that the labels of the quark partons are arbitrary to shuffle the particles around. If after the shuffling, the flavor content of a combination is identical to the one considered before, the combination is ignored. For example, consider $u \bar{d} q \bar{q}$ component of a $\pi^{+}$particle. The $q \bar{q}$ can either couple to $I=1$ or $I=0$. It turns out that the combination coupled to $I=1$ is not independent after reshuffling of the particle label.

All the hadrons are color neutral. Therefore, we couple all partons to color singlet. All possible ways of making the coupling must be considered. 


\section{Parity}

Consider a hadron moving in the z-direction with helicity $\Lambda,|P \Lambda\rangle$. Under parity transformation, the momentum changes the direction, and the helicity changes sign. However, if we make an additional $180^{\circ}$ rotation around the $y$ axis, the original momentum is restored, and we have a state $|P-\Lambda\rangle$. According to Jacob and Wick [22], we have,

$$
(-1)^{s-\Lambda} \eta|P-\Lambda\rangle=\hat{Y}|P \Lambda\rangle
$$

where $\hat{Y}$ is a parity operation followed by a $180^{\circ}$ rotation around the $y$ axis, and $\eta$ is the intrinsic parity of the hadron.

For a particle state with non-zero helicity, the above equation allows one to obtain the wave function of the state with helicity $(-\Lambda)$ from that with helicity $\Lambda$. On the other hand, for a particle of zero helicity, the above equation can be considered as a constraint on the wave function.

When $\hat{Y}$ acts on the individual partons, the transformation is

$$
(-1)^{s-\lambda} \eta\left|k_{x},-k_{y}, k_{z},-\lambda\right\rangle=\hat{Y}\left|k_{x}, k_{y}, k_{z}, \lambda\right\rangle
$$

where the intrinsic parity for a quark is +1 , an antiquark -1 , and a gluon -1 . For instance, for a $u$ quark state,

$$
\hat{Y}\left|u_{\uparrow}\right\rangle=\left|u_{\downarrow}\right\rangle, \quad \hat{Y}\left|u_{\downarrow}\right\rangle=-\left|u_{\uparrow}\right\rangle,
$$

where we have omitted the momentum label. For a $\bar{d}$ quark state,

$$
\hat{Y}\left|\bar{d}_{\uparrow}\right\rangle=-\left|\bar{d}_{\downarrow}\right\rangle, \quad \hat{Y}\left|\bar{d}_{\downarrow}\right\rangle=\left|\bar{d}_{\uparrow}\right\rangle
$$

because of the opposite intrinsic parity.

\section{E. Time Reversal}

Time reversal usually provides constraint on reality of the wave function amplitudes. Under the transformation, however, the light-cone time and coordinate interchange. To preserve the original light-cone coordinates, we consider the combined time-reversal and parity.

The light-cone gauge condition is invariant under the combined transformation. However, $A^{+}=0$ does not fix the gauge freedom completely, additional gauge fixing must be specified. Physically the additional gauge fixing corresponds to a choice of boundary conditions for gauge fields at $\xi^{-}= \pm \infty$. If one chooses the antisymmetric boundary condition, $A_{\perp}\left(\xi^{-}=\right.$ $-\infty)=-A_{\perp}\left(\xi^{-}=\infty\right)$, which is invariant under the combined transformation, then one can show that all light-cone wave function amplitudes are real (principal-value prescription). On the other hand, if one chooses either advanced or retarded boundary conditions $A_{\perp}\left(\xi^{-}=\right.$ $\pm \infty)=0$, the combined transformation is broken, and the wave function amplitudes are complex. 


\section{WAVE-FUNCTION AMPLITUDES FOR THE PION}

In this section, we classify the light-cone wave-function amplitudes for the $\pi^{+}$meson up to and including four partons. The amplitudes for other isotriplet members can be obtained by using the isospin lowering operator. The pion is a pseudoscalar meson with spin $J=0$ and parity $P=-1$. These quantum numbers are necessary constraints when the light-cone wave function amplitudes are constructed.

A pion moving in the $z$ direction has the following transformation under $\hat{Y}$,

$$
\hat{Y}\left|\pi^{+}\right\rangle=-\left|\pi^{+}\right\rangle \text {. }
$$

Every Fock component we write down must have this symmetry.

In the following subsections, we present the wave-function amplitudes of $\pi^{+}$up to fourparticle component Fock states, i.e., $u \bar{d}, u \bar{d} g, u \bar{d} g g$, and $u \bar{d} q \bar{q}$. Related studies on the light-cone distribution amplitudes for $\pi$ mesons can be found in [14, 23, 24, 25].

\section{A. The $u \bar{d}$ Component}

For this component, $n=2$, and the total quark helicity $\lambda$ can be either 0 or 1 . The isospin does not provide any additional constraint. From Eq. (7) we can have two wave-function amplitudes, corresponding to $l_{z}=0$ and $\left|l_{z}\right|=1$. They have been discussed in [3] and many other references before. For completeness, we present the results here,

$$
\begin{aligned}
\left|\pi^{+}\right\rangle_{u \bar{d}}^{l_{z}=0} & =\int d[1] d[2] \psi_{u \bar{d}}^{(1)}(1,2) \frac{\delta_{i j}}{\sqrt{3}}\left[u_{\uparrow i}^{\dagger}(1) \bar{d}_{\downarrow j}^{\dagger}(2)-u_{\downarrow i}^{\dagger}(1) \bar{d}_{\uparrow j}^{\dagger}(2)\right]|0\rangle \\
\left|\pi^{+}\right\rangle_{u \bar{d}}^{\left|l_{z}\right|=1} & =\int d[1] d[2] \psi_{u \bar{d}}^{(2)}(1,2) \frac{\delta_{i j}}{\sqrt{3}}\left[k_{1 \perp}^{-} u_{\uparrow i}^{\dagger}(1) \bar{d}_{\uparrow j}^{\dagger}(2)+k_{1 \perp}^{+} u_{\downarrow i}^{\dagger}(1) \bar{d}_{\downarrow j}^{\dagger}(2)\right]|0\rangle,
\end{aligned}
$$

where $i$ and $j=1,2,3$ are the color indices, and $\uparrow$ and $\downarrow$ label quark light-cone helicity $+1 / 2$ and $-1 / 2$, respectively. The color factor $\delta_{i j} / \sqrt{3}$ is normalized to 1 . The amplitudes

$\psi_{u \bar{d}}^{(1,2)}(1,2)$ are functions of quark momenta with argument 1 representing $x_{1}$ and $k_{1 \perp}$ and so on. The dependence on the transverse momenta is of form $\vec{k}_{i \perp} \cdot \vec{k}_{j \perp}$ only. Since the momentum conservation implies $\vec{k}_{1 \perp}+\vec{k}_{2 \perp}=0$ and $x_{1}+x_{2}=1, \psi_{u \bar{d}}^{(1,2)}(1,2)$ depend on variables $x_{1}$ and $k_{1 \perp}^{2}$ only. The integration in the above equation become,

$$
\int d[1] d[2]=\int \frac{d^{2} k_{1 \perp}}{(2 \pi)^{3}} \frac{d x_{1}}{2 \sqrt{x_{1}\left(1-x_{1}\right)}} .
$$

It is easy to check that the amplitudes $\psi_{u \bar{d}}^{(1,2)}(1,2)$ have the correct transformation behavior under $\hat{Y}$.

\section{B. The $u \bar{d} g$ Component}

For this component, $n=3$, and total parton helicity $\lambda$ can be 0,1 , or 2 . Therefore, the light-cone wave function amplitudes must have $\left|l_{z}\right|=0,1$, or 2 . Again isospin symmetry does not provide any constraint. 
To satisfy the constraint from parity, we consider the $u \bar{d}$ pair with definite properties under the $\hat{Y}$ transformation

$$
\begin{aligned}
(u \bar{d})_{S, 0}^{\dagger} & =u_{\uparrow i}^{\dagger}(1) \bar{d}_{\downarrow j}^{\dagger}(2)+u_{\downarrow i}^{\dagger}(1) \bar{d}_{\uparrow j}^{\dagger}(2), \\
(u \bar{d})_{A, 0}^{\dagger} & =u_{\uparrow i}^{\dagger}(1) \bar{d}_{\downarrow j}^{\dagger}(2)-u_{\downarrow i}^{\dagger}(1) \bar{d}_{\uparrow j}^{\dagger}(2), \\
(u \bar{d})_{A, 1}^{\dagger} & =u_{\uparrow i}^{\dagger}(1) \bar{d}_{\uparrow j}^{\dagger}(2), \\
(u \bar{d})_{A,-1}^{\dagger} & =u_{\downarrow i}^{\dagger}(1) \bar{d}_{\downarrow j}^{\dagger}(2) .
\end{aligned}
$$

It is clear that

$$
\hat{Y}(u \bar{d})_{S, \lambda_{z}}^{\dagger}|0\rangle=(u \bar{d})_{S,-\lambda_{z}}^{\dagger}|0\rangle, \quad \hat{Y}(u \bar{d})_{A, \lambda_{z}}^{\dagger}|0\rangle=-(u \bar{d})_{A,-\lambda_{z}}^{\dagger}|0\rangle .
$$

where we have neglected the transformation of the momentum labels. On the other hand, the one-gluon state transforms under $\hat{Y}$

$$
\hat{Y}\left|g_{\lambda}\right\rangle=-\left|g_{-\lambda}\right\rangle \text {. }
$$

because the gluon is a vector particle.

There is only one way to couple the color indices. The quark and anti-quark (with color indices $i$ and $j$ ) couple to an octet which in turn couples to the octet gluon (with color index a) to yield a singlet. The coupling can be achieved with an $\mathrm{SU}(3)$ matrices $T_{i j}^{a}$.

When $l_{z}=0$, the helicity of the quarks must be $\lambda_{u \bar{d}}= \pm 1$ because $\lambda_{g}=\mp 1$. From Eq. (17) we have two independent amplitudes,

$$
\begin{aligned}
& \left|\pi^{+}\right\rangle_{u \bar{d} g}^{l_{z}=0}=\int d[1] d[2] d[3] \frac{T_{i j}^{a}}{2}\left\{\psi_{u \bar{d} g}^{(1)}(1,2,3)\left[(u \bar{d})_{A, 1}^{\dagger} g_{\downarrow}^{a \dagger}(3)-(u \bar{d})_{A,-1}^{\dagger} g_{\uparrow}^{a \dagger}(3)\right]\right. \\
& \left.+i \epsilon^{\alpha \beta} k_{1 \alpha} k_{2 \beta} \psi_{u \bar{d} g}^{(2)}(1,2,3)\left[(u \bar{d})_{A, 1}^{\dagger} g_{\downarrow}^{a \dagger}(3)+(u \bar{d})_{A,-1}^{\dagger} g_{\uparrow}^{a \dagger}(3)\right]\right\}|0\rangle,
\end{aligned}
$$

where $\alpha, \beta=x, y$ are the transverse indices. Again the color factor $T_{i j}^{a} / 2$ is normalized to unit. The above state obey the right transformation under $\hat{Y}$ because the wave-function amplitudes are invariant when all $y$-components of the parton momenta change sign.

When $\left|l_{z}\right|=1$, the total quark helicity must be $\lambda_{u \bar{d}}=0$ again because $\lambda_{g}= \pm 1$. We can write down 4 independent wave-function amplitudes,

$$
\begin{aligned}
\left|\pi^{+}\right\rangle_{u \bar{d} g}^{\left|l_{z}\right|=1}= & \int d[1] d[2] d[3] \frac{T_{i j}^{a}}{2}\left\{\psi_{u \bar{d} g}^{(3)}(1,2,3)\left[k_{1 \perp}^{+}(u \bar{d})_{A, 0}^{\dagger} g_{\downarrow}^{a \dagger}(3)-k_{1 \perp}^{-}(u \bar{d})_{A, 0}^{\dagger} g_{\uparrow}^{a \dagger}(3)\right]\right. \\
& +\psi_{u \bar{d} g}^{(4)}(1,2,3)\left[k_{2 \perp}^{+}(u \bar{d})_{A, 0}^{\dagger} g_{\downarrow}^{a \dagger}(3)-k_{2 \perp}^{-}(u \bar{d})_{A, 0}^{\dagger} g_{\uparrow}^{a \dagger}(3)\right] \\
& +\psi_{u \bar{d} g}^{(5)}(1,2,3)\left[k_{1 \perp}^{+}(u \bar{d})_{S, 0}^{\dagger} g_{\downarrow}^{a \dagger}(3)+k_{1 \perp}^{-}(u \bar{d})_{S, 0}^{\dagger} g_{\uparrow}^{a \dagger}(3)\right] \\
& \left.+\psi_{u \bar{d} g}^{(6)}(1,2,3)\left[k_{2 \perp}^{+}(u \bar{d})_{S, 0}^{\dagger} g_{\downarrow}^{a \dagger}(3)+k_{2 \perp}^{-}(u \bar{d})_{S, 0}^{\dagger} g_{\uparrow}^{a \dagger}(3)\right]\right\}|0\rangle .
\end{aligned}
$$

Finally, when $\left|l_{z}\right|=2$, the total quark helicity $\lambda_{u \bar{d}}= \pm 1$, and $\lambda_{g}= \pm 1$. We have 3 amplitudes

$$
\begin{aligned}
\left|\pi^{+}\right\rangle_{u \bar{d} g}^{\left|l_{z}\right|=2}= & \int d[1] d[2] d[3] \frac{T_{i j}^{a}}{2}\left\{\psi_{u \bar{d} g}^{(7)}(1,2,3)\left[k_{1 \perp}^{+} k_{1 \perp}^{+}(u \bar{d})_{A,-1}^{\dagger} g_{\downarrow}^{a \dagger}(3)-k_{1 \perp}^{-} k_{1 \perp}^{-}(u \bar{d})_{A, 1}^{\dagger} g_{\uparrow}^{a \dagger}(3)\right]\right. \\
& +\psi_{u \bar{d} g}^{(8)}(1,2,3)\left[k_{1 \perp}^{+} k_{2 \perp}^{+}(u \bar{d})_{A,-1}^{\dagger} g_{\downarrow}^{a \dagger}(3)-k_{1 \perp}^{-} k_{2 \perp}^{-}(u \bar{d})_{A, 1}^{\dagger} g_{\uparrow}^{a \dagger}(3)\right] \\
& \left.+\psi_{u \bar{d} g}^{(9)}(1,2,3)\left[k_{2 \perp}^{+} k_{2 \perp}^{+}(u \bar{d})_{A,-1}^{\dagger} g_{\downarrow}^{a \dagger}(3)-k_{2 \perp}^{-} k_{2 \perp}^{-}(u \bar{d})_{A, 1}^{\dagger} g_{\uparrow}^{a \dagger}(3)\right]\right\}|0\rangle
\end{aligned}
$$


Summing up, we have a total of 9 independent light-cone amplitudes for the pion component with 3 partons.

\section{The $u \bar{d} g g$ Component}

For this component, the helicity of the two-gluons can be $\lambda_{g g}=0, \pm 2$, and that for quarks $\lambda_{u \bar{d}}=0, \pm 1$, and so $\left|l_{z}\right|=0,1,2$, or 3 . To make the $\hat{Y}$ transformation simple, we combine the two gluons in the similar way as we did for $u \bar{d}$ in the last subsection,

$$
\begin{aligned}
(g g)_{S, 0}^{\dagger} & =g_{\uparrow a}^{\dagger}(3) g_{\downarrow b}^{\dagger}(4)+g_{\downarrow a}^{\dagger}(3) g_{\uparrow b}^{\dagger}(4), \\
(g g)_{A, 0}^{\dagger} & =g_{\uparrow a}^{\dagger}(3) g_{\downarrow b}^{\dagger}(4)-g_{\downarrow a}^{\dagger}(3) g_{\uparrow b}^{\dagger}(4), \\
(g g)_{S, 2}^{\dagger} & =g_{\uparrow a}^{\dagger}(3) g_{\uparrow b}^{\dagger}(4), \\
(g g)_{S,-2}^{\dagger} & =g_{\downarrow a}^{\dagger}(3) g_{\downarrow b}^{\dagger}(4),
\end{aligned}
$$

where the subscripts $A$ and $S$ indicate that there is a factor -1 and 1 , respectively, under the $\hat{Y}$ transformation.

There are three different ways to couple the color indices of the two quarks and two gluons to form color-singlets. If the color indices for the two quarks are $i$ and $j$ and those for two gluons are $a$ and $b$, we have the singlet combinations: $f_{a b c} T_{i j}^{c}, d_{a b c} T_{i j}^{c}$, and $\delta_{a b} \delta_{i j}$. The last two are symmetric in the color indices of the two gluons, while the first one is antisymmetric. In the following, we only present the results for the color coupling $\delta_{a b} \delta_{i j}$ (the quark pair and two gluons are both color-singlet), and those for the other two couplings can be obtained similarly.

To maximally utilize Bose symmetry between the two gluons, we will eliminate the momentum of the up quark (labeled by 1 below) in favor of the momenta of anti-down quark and the two gluons.

For $l_{z}=0$, the only possible parton helicity combination is $\lambda_{g g}=0$ and $\lambda_{u \bar{d}}=0$. In this case, we have 6 independent light-cone amplitudes following Eq. (17),

$$
\begin{aligned}
\left|\pi^{+}\right\rangle_{u \bar{d} g g}^{l_{z}=0}= & \int d[1] d[2] d[3] d[4] \frac{\delta_{i j} \delta^{a b}}{\sqrt{24}}\left\{\psi_{u \bar{d} g g}^{(1)}(1,2,3,4)(u \bar{d})_{A, 0}^{\dagger}(g g)_{S, 0}^{\dagger}\right. \\
& +\psi_{u \bar{d} g g}^{(2)}(1,2,3,4)(u \bar{d})_{S, 0}^{\dagger}(g g)_{A, 0}^{\dagger} \\
& +i \epsilon^{\alpha \beta} k_{2 \alpha} k_{3 \beta} \psi_{u \bar{d} g g}^{(3)}(1,2,3,4)(u \bar{d})_{S, 0}^{\dagger}(g g)_{S, 0}^{\dagger} \\
& +i \epsilon^{\alpha \beta} k_{3 \alpha} k_{4 \beta} \psi_{u \bar{d} g g}^{(4)}(1,2,3,4)(u \bar{d})_{S, 0}^{\dagger}(g g)_{S, 0}^{\dagger} \\
& +i \epsilon^{\alpha \beta} k_{2 \alpha} k_{3 \beta} \psi_{u \bar{d} g g}^{(5)}(1,2,3,4)(u \bar{d})_{A, 0}^{\dagger}(g g)_{A, 0}^{\dagger} \\
& \left.+i \epsilon^{\alpha \beta} k_{3 \alpha} k_{4 \beta} \psi_{u \bar{d} g g}^{(6)}(1,2,3,4)(u \bar{d})_{A, 0}^{\dagger}(g g)_{A, 0}^{\dagger}\right\}|0\rangle
\end{aligned}
$$

where we have used the symmetry between two gluons $(3 \leftrightarrow 4)$ to reduce the number of independent amplitudes. For example, $i \epsilon^{\alpha \beta} k_{2 \alpha} k_{4 \beta}$ can be obtained from $i \epsilon^{\alpha \beta} k_{2 \alpha} k_{3 \beta}$ by 3 and 4 exchange, and so the former is not independent. This property is general for all of the lightcone amplitudes of $u \bar{d} g g$ component, and will be used throughout the following classification. Because of (anti)symmetric properties for the two gluons, the above amplitudes have the following symmetry: $\psi_{u \bar{d} g g}^{(1,6)}(1,2,3,4)=\psi_{u \bar{d} g g}^{(1,6)}(1,2,4,3)$ and $\psi_{u \bar{d} g g}^{(2,4)}(1,2,3,4)=-\psi_{u \bar{d} g g}^{(2,4)}(1,2,4,3)$. 
For $\left|l_{z}\right|=1$, the parton helicity has two possible combinations: either $\lambda_{g g}=0$ and $\lambda_{u \bar{d}}=\mp 1$, or $\lambda_{g g}=\mp 2$ and $\lambda_{u \bar{d}}= \pm 1$. For the first case, we have 8 independent amplitudes,

$$
\begin{aligned}
\left|\pi^{+}\right\rangle_{u \bar{d} g g}^{\left|l_{z}\right|=1}= & \int d[1] d[2] d[3] d[4] \frac{\delta^{i j} \delta_{a b}}{\sqrt{24}}\{ \\
& \psi_{u \bar{d} g g}^{(7)}(1,2,3,4)\left[k_{2 \perp}^{+}(u \bar{d})_{A,-1}^{\dagger}(g g)_{S, 0}^{\dagger}+k_{2 \perp}^{-}(u \bar{d})_{A, 1}^{\dagger}(g g)_{S, 0}^{\dagger}\right] \\
& +\psi_{u \bar{d} g g}^{(8)}(1,2,3,4)\left[k_{3 \perp}^{+}(u \bar{d})_{A,-1}^{\dagger}(g g)_{S, 0}^{\dagger}+k_{3 \perp}^{-}(u \bar{d})_{A, 1}^{\dagger}(g g)_{S, 0}^{\dagger}\right] \\
& +\psi_{u \bar{d} g g}^{(9)}(1,2,3,4)\left[k_{2 \perp}^{+}(u \bar{d})_{A,-1}^{\dagger}(g g)_{A, 0}^{\dagger}-k_{2 \perp}^{-}(u \bar{d})_{A, 1}^{\dagger}(g g)_{A, 0}^{\dagger}\right] \\
& +\psi_{u \bar{d} g g}^{(10)}(1,2,3,4)\left[k_{3 \perp}^{+}(u \bar{d})_{A,-1}^{\dagger}(g g)_{A, 0}^{\dagger}-k_{3 \perp}^{-}(u \bar{d})_{A, 1}^{\dagger}(g g)_{A, 0}^{\dagger}\right] \\
& +i \epsilon^{\alpha \beta} k_{3 \alpha} k_{4 \beta} \psi_{u \bar{d} g g}^{(11)}(1,2,3,4)\left[k_{2 \perp}^{+}(u \bar{d})_{A,-1}^{\dagger}(g g)_{S, 0}^{\dagger}-k_{2 \perp}^{-}(u \bar{d})_{A, 1}^{\dagger}(g g)_{S, 0}^{\dagger}\right] \\
& +i \epsilon^{\alpha \beta} k_{2 \alpha} k_{4 \beta} \psi_{u \bar{d} g g}^{(12)}(1,2,3,4)\left[k_{3 \perp}^{+}(u \bar{d})_{A,-1}^{\dagger}(g g)_{S, 0}^{\dagger}-k_{3 \perp}^{-}(u \bar{d})_{A, 1}^{\dagger}(g g)_{S, 0}^{\dagger}\right] \\
& +i \epsilon^{\alpha \beta} k_{3 \alpha} k_{4 \beta} \psi_{u \bar{d} g g}^{(13)}(1,2,3,4)\left[k_{2 \perp}^{+}(u \bar{d})_{A,-1}^{\dagger}(g g)_{A, 0}^{\dagger}+k_{2 \perp}^{-}(u \bar{d})_{A, 1}^{\dagger}(g g)_{A, 0}^{\dagger}\right] \\
& \left.+i \epsilon^{\alpha \beta} k_{2 \alpha} k_{4 \beta} \psi_{u \bar{d} g g}^{(14)}(1,2,3,4)\left[k_{3 \perp}^{+}(u \bar{d})_{A,-1}^{\dagger}(g g)_{A, 0}^{\dagger}+k_{3 \perp}^{-}(u \bar{d})_{A, 1}^{\dagger}(g g)_{A, 0}^{\dagger}\right]\right\}|0\rangle,
\end{aligned}
$$

where the $3 \leftrightarrow 4$ symmetry again plays an important role to reduce the number of independent amplitudes. For the second case, $\lambda_{g g}=\mp 2$ and $\lambda_{u \bar{d}}= \pm 1$, we find 4 independent amplitudes:

$$
\begin{aligned}
\left|\pi^{+}\right\rangle_{u \bar{d} g g}^{\left|l_{z}\right|=1}= & \int d[1] d[2] d[3] d[4] \frac{\delta^{i j} \delta_{a b}}{\sqrt{24}}\{ \\
& \psi_{u \bar{d} g g}^{(15)}(1,2,3,4)\left[k_{2 \perp}^{+}(u \bar{d})_{A, 1}^{\dagger}(g g)_{S,-2}^{\dagger}+k_{2 \perp}^{-}(u \bar{d})_{A,-1}^{\dagger}(g g)_{S, 2}^{\dagger}\right] \\
& +\psi_{u \bar{d} g g}^{(16)}(1,2,3,4)\left[k_{3 \perp}^{+}(u \bar{d})_{A, 1}^{\dagger}(g g)_{S,-2}^{\dagger}+k_{3 \perp}^{-}(u \bar{d})_{A,-1}^{\dagger}(g g)_{S, 2}^{\dagger}\right] \\
& +i \epsilon^{\alpha \beta} k_{3 \alpha} k_{4 \beta} \psi_{u \bar{d} g g}^{(17)}(1,2,3,4)\left[k_{2 \perp}^{+}(u \bar{d})_{A, 1}^{\dagger}(g g)_{S,-2}^{\dagger}-k_{2 \perp}^{-}(u \bar{d})_{A,-1}^{\dagger}(g g)_{S, 2}^{\dagger}\right] \\
& \left.+i \epsilon^{\alpha \beta} k_{3 \alpha} k_{4 \beta} \psi_{u \bar{d} g g}^{(18)}(1,2,3,4)\left[k_{3 \perp}^{+}(u \bar{d})_{A, 1}^{\dagger}(g g)_{S,-2}^{\dagger}-k_{3 \perp}^{-}(u \bar{d})_{A,-1}^{\dagger}(g g)_{S, 2}^{\dagger}\right]\right\}|0\rangle .
\end{aligned}
$$

The Bose symmetry implies the following constraints: $\psi_{u \bar{d} g g}^{(7,13,15)}(1,2,3,4)=$ $\psi_{u \bar{d} g g}^{(7,13,15)}(1,2,4,3)$, and $\psi_{u \bar{d} g g}^{(8,11,17)}(1,2,3,4)=-\psi_{u \bar{d} g g}^{(8,11,17)}(1,2,4,3)$.

For $\left|l_{z}\right|=2$, the parton helicity must be $\lambda_{g g}=\mp 2$ and $\lambda_{u \bar{d}}=0$. We find 12 independent amplitudes

$$
\begin{aligned}
\left|\pi^{+}\right\rangle_{u \bar{d} g g}^{\left|l_{z}\right|=2}= & \int d[1] d[2] d[3] d[4] \frac{\delta^{i j} \delta_{a b}}{\sqrt{24}}\{ \\
& \psi_{u \bar{d} g g}^{(19)}(1,2,3,4)\left[k_{2 \perp}^{+} k_{2 \perp}^{+}(u \bar{d})_{A, 0}^{\dagger}(g g)_{S,-2}^{\dagger}+k_{2 \perp}^{-} k_{2 \perp}^{-}(u \bar{d})_{A, 0}^{\dagger}(g g)_{S, 2}^{\dagger}\right] \\
& +\psi_{u \bar{d} g g}^{(20)}(1,2,3,4)\left[k_{2 \perp}^{+} k_{3 \perp}^{+}(u \bar{d})_{A, 0}^{\dagger}(g g)_{S,-2}^{\dagger}+k_{2 \perp}^{-} k_{3 \perp}^{-}(u \bar{d})_{A, 0}^{\dagger}(g g)_{S, 2}^{\dagger}\right] \\
& +\psi_{u \bar{d} g g}^{(21)}(1,2,3,4)\left[k_{3 \perp}^{+} k_{3 \perp}^{+}(u \bar{d})_{A, 0}^{\dagger}(g g)_{S,-2}^{\dagger}+k_{3 \perp}^{-} k_{3 \perp}^{-}(u \bar{d})_{A, 0}^{\dagger}(g g)_{S, 2}^{\dagger}\right] \\
& +\psi_{u \bar{d} g g}^{(22)}(1,2,3,4)\left[k_{3 \perp}^{+} k_{4 \perp}^{+}(u \bar{d})_{A, 0}^{\dagger}(g g)_{S,-2}^{\dagger}+k_{3 \perp}^{-} k_{4 \perp}^{-}(u \bar{d})_{A, 0}^{\dagger}(g g)_{S, 2}^{\dagger}\right]
\end{aligned}
$$




$$
\begin{aligned}
& +i \epsilon^{\alpha \beta} k_{3 \alpha} k_{4 \beta} \psi_{u \bar{d} g g}^{(23)}(1,2,3,4)\left[k_{2 \perp}^{+} k_{2 \perp}^{+}(u \bar{d})_{A, 0}^{\dagger}(g g)_{S,-2}^{\dagger}-k_{2 \perp}^{-} k_{2 \perp}^{-}(u \bar{d})_{A, 0}^{\dagger}(g g)_{S, 2}^{\dagger}\right] \\
& +i \epsilon^{\alpha \beta} k_{2 \alpha} k_{4 \beta} \psi_{u \bar{d} g g}^{(24)}(1,2,3,4)\left[k_{3 \perp}^{+} k_{3 \perp}^{+}(u \bar{d})_{A, 0}^{\dagger}(g g)_{S,-2}^{\dagger}-k_{3 \perp}^{-} k_{3 \perp}^{-}(u \bar{d})_{A, 0}^{\dagger}(g g)_{S, 2}^{\dagger}\right] \\
& +\psi_{u \bar{d} g g}^{(25)}(1,2,3,4)\left[k_{2 \perp}^{+} k_{2 \perp}^{+}(u \bar{d})_{S, 0}^{\dagger}(g g)_{S,-2}^{\dagger}-k_{2 \perp}^{-} k_{2 \perp}^{-}(u \bar{d})_{S, 0}^{\dagger}(g g)_{S, 2}^{\dagger}\right] \\
& +\psi_{u \bar{d} g g}^{(26)}(1,2,3,4)\left[k_{2 \perp}^{+} k_{3 \perp}^{+}(u \bar{d})_{S, 0}^{\dagger}(g g)_{S,-2}^{\dagger}-k_{2 \perp}^{-} k_{3 \perp}^{-}(u \bar{d})_{S, 0}^{\dagger}(g g)_{S, 2}^{\dagger}\right] \\
& +\psi_{u \bar{d} g g}^{(27)}(1,2,3,4)\left[k_{3 \perp}^{+} k_{3 \perp}^{+}(u \bar{d})_{S, 0}^{\dagger}(g g)_{S,-2}^{\dagger}-k_{3 \perp}^{-} k_{3 \perp}^{-}(u \bar{d})_{S, 0}^{\dagger}(g g)_{S, 2}^{\dagger}\right] \\
& +\psi_{u \bar{d} g g}^{(28)}(1,2,3,4)\left[k_{3 \perp}^{+} k_{4 \perp}^{+}(u \bar{d})_{S, 0}^{\dagger}(g g)_{S,-2}^{\dagger}-k_{3 \perp}^{-} k_{4 \perp}^{-}(u \bar{d})_{S, 0}^{\dagger}(g g)_{S, 2}^{\dagger}\right] \\
& +i \epsilon^{\alpha \beta} k_{3 \alpha} k_{4 \beta} \psi_{u \bar{d} g g}^{(29)}(1,2,3,4)\left[k_{2 \perp}^{+} k_{2 \perp}^{+}(u \bar{d})_{S, 0}^{\dagger}(g g)_{S,-2}^{\dagger}+k_{2 \perp}^{-} k_{2 \perp}^{-}(u \bar{d})_{S, 0}^{\dagger}(g g)_{S, 2}^{\dagger}\right] \\
& \left.+i \epsilon^{\alpha \beta} k_{2 \alpha} k_{4 \beta} \psi_{u \bar{d} g g}^{(30)}(1,2,3,4)\left[k_{3 \perp}^{+} k_{3 \perp}^{+}(u \bar{d})_{S, 0}^{\dagger}(g g)_{S,-2}^{\dagger}+k_{3 \perp}^{-} k_{3 \perp}^{-}(u \bar{d})_{S, 0}^{\dagger}(g g)_{S, 2}^{\dagger}\right]\right\}|0\rangle .
\end{aligned}
$$

The Bose symmetry yields the following constraints: $\quad \psi_{u \bar{d} g g}^{(19,22,25,28)}(1,2,3,4)=$ $\psi_{u \bar{d} g g}^{(19,22,25,28)}(1,2,4,3)$ and $\psi_{u \bar{d} g g}^{(23,29)}(1,2,3,4)=-\psi_{u \bar{d} g g}^{(23,29)}(1,2,4,3)$.

For $\left|l_{z}\right|=3$, the parton helicity must be $\lambda_{g g}= \pm 2$ and $\lambda_{u \bar{d}}= \pm 1$. We find 8 independent light-cone amplitudes

$$
\begin{aligned}
\left|\pi^{+}\right\rangle_{u \bar{d} g g}^{\left|l_{z}\right|=3}= & \int d[1] d[2] d[3] d[4] \frac{\delta^{i j} \delta_{a b}}{\sqrt{24}}\{ \\
& \psi_{u \bar{d} g g}^{(31)}(1,2,3,4)\left[\left(k_{2 \perp}^{+}\right)^{3}(u \bar{d})_{A,-1}^{\dagger}(g g)_{S,-2}^{\dagger}+\left(k_{2 \perp}^{-}\right)^{3}(u \bar{d})_{A, 1}^{\dagger}(g g)_{S, 2}^{\dagger}\right] \\
& +\psi_{u \bar{d} g g}^{(32)}(1,2,3,4)\left[\left(k_{3 \perp}^{+}\right)^{3}(u \bar{d})_{A,-1}^{\dagger}(g g)_{S,-2}^{\dagger}+\left(k_{3 \perp}^{-}\right)^{3}(u \bar{d})_{A, 1}^{\dagger}(g g)_{S, 2}^{\dagger}\right] \\
& +\psi_{u \bar{d} g g}^{(33)}(1,2,3,4)\left[\left(k_{2 \perp}^{+}\right)^{2} k_{3 \perp}^{+}(u \bar{d})_{A,-1}^{\dagger}(g g)_{S,-2}^{\dagger}+\left(k_{2 \perp}^{-}\right)^{2} k_{3 \perp}^{-}(u \bar{d})_{A, 1}^{\dagger}(g g)_{S, 2}^{\dagger}\right] \\
& +\psi_{u \bar{d} g g}^{(34)}(1,2,3,4)\left[\left(k_{3 \perp}^{+}\right)^{2} k_{2 \perp}^{+}(u \bar{d})_{A,-1}^{\dagger}(g g)_{S,-2}^{\dagger}+\left(k_{3 \perp}^{-}\right)^{2} k_{2 \perp}^{-}(u \bar{d})_{A, 1}^{\dagger}(g g)_{S, 2}^{\dagger}\right] \\
& +\psi_{u \bar{d} g g}^{(35)}(1,2,3,4)\left[\left(k_{3 \perp}^{+}\right)^{2} k_{4 \perp}^{+}(u \bar{d})_{A,-1}^{\dagger}(g g)_{S,-2}^{\dagger}+\left(k_{3 \perp}^{-}\right)^{2} k_{4 \perp}^{-}(u \bar{d})_{A, 1}^{\dagger}(g g)_{S, 2}^{\dagger}\right] \\
& +\psi_{u \bar{d} g g}^{(36)}(1,2,3,4)\left[k_{2 \perp}^{+} k_{3 \perp}^{+} k_{4 \perp}^{+}(u \bar{d})_{A,-1}^{\dagger}(g g)_{S,-2}^{\dagger}+k_{2 \perp}^{-} k_{3 \perp}^{-} k_{4 \perp}^{-}(u \bar{d})_{A, 1}^{\dagger}(g g)_{S, 2}^{\dagger}\right] \\
& +i \epsilon^{\alpha \beta} k_{3 \alpha} k_{4 \beta} \psi_{u \bar{d} g g}^{(37)}(1,2,3,4)\left[\left(k_{2 \perp}^{+}\right)^{3}(u \bar{d})_{A,-1}^{\dagger}(g g)_{S,-2}^{\dagger}-\left(k_{2 \perp}^{-}\right)^{3}(u \bar{d})_{A, 1}^{\dagger}(g g)_{S, 2}^{\dagger}\right] \\
& \left.+i \epsilon^{\alpha \beta} k_{2 \alpha} k_{4 \beta} \psi_{u \bar{d} g g}^{(38)}(1,2,3,4)\left[\left(k_{3 \perp}^{+}\right)^{3}(u \bar{d})_{A,-1}^{\dagger}(g g)_{S,-2}^{\dagger}-\left(k_{3 \perp}^{-}\right)^{3}(u \bar{d})_{A, 1}^{\dagger}(g g)_{S, 2}^{\dagger}\right]\right\}|0\rangle .
\end{aligned}
$$

The Bose symmetry implies the symmetry relations: $\psi_{u \bar{d} g g}^{(31,36)}(1,2,3,4)=\psi_{u \bar{d} g g}^{(31,36)}(1,2,4,3)$ and $\psi_{u \bar{d} g g}^{(37)}(1,2,3,4)=-\psi_{u \bar{d} g g}^{(37)}(1,2,4,3)$.

Similarly, one can obtain the amplitudes when the quark pair and two gluons are in coloroctet states. If the two gluons are symmetric in color, we have $\psi_{u \bar{d} g g}^{(i)}$ with $i=39, \cdots, 76$, defined in the same way as the above equations except the color factor is replaced by $\sqrt{\frac{3}{20}} d_{a b c} T_{i j}^{c}$. When the two gluons are antisymmetric in color, we obtain $\psi_{u \bar{d} g g}^{(i)}$ with $i=77, \cdots, 114$, again defined in the same way, except with the color factor $\sqrt{\frac{1}{12}} f_{a b c} T_{i j}^{c}$. Note that there are sign changes for the symmetry relations derived from Bose symmetry. 
Therefore, we have a total of $38 \times 3=114$ independent amplitudes for the Fock component $u \bar{d} g g$ in $\pi^{+}$.

\section{The $u \bar{d} q \bar{q}$ Component}

We first consider the up and down sea-quark flavors. In this case, the following two flavor structures have total isospin $I=1$ :

$$
\begin{aligned}
& u \bar{d}(u \bar{u}+d \bar{d}) \\
& (u \bar{u}-d \bar{d}) u \bar{d}-u \bar{d}(u \bar{u}-d \bar{d}) .
\end{aligned}
$$

The first structure arises from the first quark pair coupled to isospin 1 and the second pair coupled to isospin 0 . The second structure comes from both pairs coupled to isospin 1. However, after some rearrangements of the particle labels, the second structure can be reduced to the first one, and hence is not independent. Therefore, we consider only the first isospin structure with all possible color and spin combinations.

To simplify the $\hat{Y}$ transformation, we introduce the following combinations for the sea quark pair:

$$
\begin{aligned}
(q \bar{q})_{S, 0}^{\dagger} & =u_{\uparrow k}^{\dagger}(3) \bar{u}_{\downarrow l}^{\dagger}(4)+u_{\downarrow k}^{\dagger}(3) \bar{u}_{\uparrow l}^{\dagger}(4)+d_{\uparrow k}^{\dagger}(3) \bar{d}_{\downarrow l}^{\dagger}(4)+d_{\downarrow k}^{\dagger}(3) \bar{d}_{\uparrow l}^{\dagger}(4), \\
(q \bar{q})_{A, 0}^{\dagger} & =u_{\uparrow k}^{\dagger}(3) \bar{u}_{\downarrow l}^{\dagger}(4)-u_{\downarrow k}^{\dagger}(3) \bar{u}_{\uparrow l}^{\dagger}(4)+d_{\uparrow k}^{\dagger}(3) \bar{d}_{\downarrow l}^{\dagger}(4)-d_{\downarrow k}^{\dagger}(3) \bar{d}_{\uparrow l}^{\dagger}(4), \\
(q \bar{q})_{A, 1}^{\dagger} & =u_{\uparrow k}^{\dagger}(3) \bar{u}_{\uparrow l}^{\dagger}(4)+d_{\uparrow k}^{\dagger}(3) \bar{d}_{\uparrow l}^{\dagger}(4) \\
(q \bar{q})_{A,-1}^{\dagger} & =u_{\downarrow k}^{\dagger}(3) \bar{u}_{\downarrow l}^{\dagger}(4)+d_{\downarrow k}^{\dagger}(3) \bar{d}_{\downarrow l}^{\dagger}(4) .
\end{aligned}
$$

We use them as basic building blocks in the Fock expansion.

We can form two color-singlet structures from the four color indices $i, j, k$, and $l: \delta_{i j} \delta_{k l}$ and $\delta_{i l} \delta_{j k}$, where we have implicitly assumed that the first and third are quarks' and second and fourth are antiquarks'. The first structure corresponds to the state in which the two quark-pairs are both coupled to color-singlet, while the second corresponds to the state in which the two quark-pairs are in color-octet. The wave-function amplitudes for both color combinations are similar.

The quark helicity has combinations $\lambda_{u \bar{d}}=0, \pm 1$, and $\lambda_{u \bar{u}+d \bar{d}}=0, \pm 1$. Therefore we can have three different orbital angular momentum projection $\left|l_{z}\right|=0,1,2$.

For $l_{z}=0$, the quark helicity has the combination $\lambda_{u \bar{d}}=0$ and $\lambda_{u \bar{u}+d \bar{d}}=0$, or $\lambda_{u \bar{d}}= \pm 1$ and $\lambda_{u \bar{u}+d \bar{d}}=\mp 1$. Together, we find 12 independent amplitudes:

$$
\begin{aligned}
\left|\pi^{+}\right\rangle_{u \bar{q} q \bar{q}}^{l_{z}=0}= & \int d[1] d[2] d[3] d[4] \frac{\delta_{i j} \delta_{k l}}{3}\{ \\
& \psi_{u \bar{q} q \bar{q}}^{(1)}(1,2,3,4)(u \bar{d})_{A, 0}^{\dagger}(q \bar{q})_{S, 0}^{\dagger}+\psi_{u \bar{d} q \bar{q}}^{(2)}(1,2,3,4)(u \bar{d})_{S, 0}^{\dagger}(q \bar{q})_{A, 0}^{\dagger} \\
& +i \epsilon^{\alpha \beta} k_{1 \alpha} k_{2 \beta} \psi_{u \bar{d} q \bar{q}}^{(3)}(1,2,3,4)(u \bar{d})_{S, 0}^{\dagger}(q \bar{q})_{S, 0}^{\dagger} \\
& +i \epsilon^{\alpha \beta} k_{1 \alpha} k_{3 \beta} \psi_{u \bar{d} q \bar{q}}^{(4)}(1,2,3,4)(u \bar{d})_{S, 0}^{\dagger}(q \bar{q})_{S, 0}^{\dagger} \\
& +i \epsilon^{\alpha \beta} k_{2 \alpha} k_{3 \beta} \psi_{u \bar{d} q \bar{q}}^{(5)}(1,2,3,4)(u \bar{d})_{S, 0}^{\dagger}(q \bar{q})_{S, 0}^{\dagger} \\
& +i \epsilon^{\alpha \beta} k_{1 \alpha} k_{2 \beta} \psi_{u \bar{d} q \bar{q}}^{(6)}(1,2,3,4)(u \bar{d})_{A, 0}^{\dagger}(q \bar{q})_{A, 0}^{\dagger}
\end{aligned}
$$




$$
\begin{aligned}
& +i \epsilon^{\alpha \beta} k_{1 \alpha} k_{3 \beta} \psi_{u \bar{d} q \bar{q}}^{(7)}(1,2,3,4)(u \bar{d})_{A, 0}^{\dagger}(q \bar{q})_{A, 0}^{\dagger} \\
& +i \epsilon^{\alpha \beta} k_{2 \alpha} k_{3 \beta} \psi_{u \bar{d} q \bar{q}}^{(8)}(1,2,3,4)(u \bar{d})_{A, 0}^{\dagger}(q \bar{q})_{A, 0}^{\dagger} \\
& +\psi_{u \bar{d} q \bar{q}}^{(9)}(1,2,3,4)\left[(u \bar{d})_{A, 1}^{\dagger}(q \bar{q})_{A,-1}^{\dagger}-(u \bar{d})_{A,-1}^{\dagger}(q \bar{q})_{A, 1}^{\dagger}\right] \\
& +i \epsilon^{\alpha \beta} k_{1 \alpha} k_{2 \beta} \psi_{u \bar{d} q \bar{q}}^{(10)}(1,2,3,4)\left[(u \bar{d})_{A, 1}^{\dagger}(q \bar{q})_{A,-1}^{\dagger}+(u \bar{d})_{A,-1}^{\dagger}(q \bar{q})_{A, 1}^{\dagger}\right] \\
& +i \epsilon^{\alpha \beta} k_{1 \alpha} k_{3 \beta} \psi_{u \bar{d} q \bar{q}}^{(11)}(1,2,3,4)\left[(u \bar{d})_{A, 1}^{\dagger}(q \bar{q})_{A,-1}^{\dagger}+(u \bar{d})_{A,-1}^{\dagger}(q \bar{q})_{A, 1}^{\dagger}\right] \\
& \left.+i \epsilon^{\alpha \beta} k_{2 \alpha} k_{3 \beta} \psi_{u \bar{d} q \bar{q}}^{(12)}(1,2,3,4)\left[(u \bar{d})_{A, 1}^{\dagger}(q \bar{q})_{A,-1}^{\dagger}+(u \bar{d})_{A,-1}^{\dagger}(q \bar{q})_{A, 1}^{\dagger}\right]\right\}|0\rangle .
\end{aligned}
$$

Note that $\delta_{i j}$ implicitly contracts the color indices in the $u \bar{d}$ pair and $\delta_{k l}$ contracts the $q \bar{q}$ pair.

For $\left|l_{z}\right|=1$, the quark helicity can either be in the combination $\lambda_{u \bar{d}}=0$ and $\lambda_{u \bar{u}}=-1$, or $\lambda_{u \bar{d}}=-1$ and $\lambda_{u \bar{u}}=0$. Taking together, we find 24 independent amplitudes:

$$
\begin{aligned}
\left|\pi^{+}\right\rangle_{u \bar{d} q \bar{q}}^{\mid z_{z}=1}= & \int d[1] d[2] d[3] d[4] \frac{\delta_{i j} \delta_{k l}}{3}\{ \\
& \psi_{u \bar{q} q \bar{q}}^{(13)}(1,2,3,4)\left[k_{1 \perp}^{+}(u \bar{d})_{A, 0}^{\dagger}(q \bar{q})_{A,-1}^{\dagger}-k_{1 \perp}^{-}(u \bar{d})_{A, 0}^{\dagger}(q \bar{q})_{A, 1}^{\dagger}\right] \\
& +\psi_{u \bar{d} q \bar{q}}^{(14)}(1,2,3,4)\left[k_{1 \perp}^{+}(u \bar{d})_{S, 0}^{\dagger}(q \bar{q})_{A,-1}^{\dagger}+k_{1 \perp}^{-}(u \bar{d})_{S, 0}^{\dagger}(q \bar{q})_{A, 1}^{\dagger}\right] \\
& +\psi_{u \bar{d} q \bar{q}}^{(15)}(1,2,3,4)\left[k_{1 \perp}^{+}(u \bar{d})_{A,-1}^{\dagger}(q \bar{q})_{A, 0}^{\dagger}-k_{1 \perp}^{-}(u \bar{d})_{A, 1}^{\dagger}(q \bar{q})_{A, 0}^{\dagger}\right] \\
& +\psi_{u \bar{d} q \bar{q}}^{(16)}(1,2,3,4)\left[k_{1 \perp}^{+}(u \bar{d})_{A,-1}^{\dagger}(q \bar{q})_{S, 0}^{\dagger}+k_{1 \perp}^{-}(u \bar{d})_{A, 1}^{\dagger}(q \bar{q})_{S, 0}^{\dagger}\right] \\
& +\psi_{u \bar{d} q \bar{q}}^{(17)}(1,2,3,4)\left[k_{2 \perp}^{+}(u \bar{d})_{A, 0}^{\dagger}(q \bar{q})_{A,-1}^{\dagger}-k_{2 \perp}^{-}(u \bar{d})_{A, 0}^{\dagger}(q \bar{q})_{A, 1}^{\dagger}\right] \\
& +\psi_{u \bar{d} q \bar{q}}^{(18)}(1,2,3,4)\left[k_{2 \perp}^{+}(u \bar{d})_{S, 0}^{\dagger}(q \bar{q})_{A,-1}^{\dagger}+k_{2 \perp}^{-}(u \bar{d})_{S, 0}^{\dagger}(q \bar{q})_{A, 1}^{\dagger}\right] \\
& +\psi_{u \bar{d} q \bar{q}}^{(19)}(1,2,3,4)\left[k_{2 \perp}^{+}(u \bar{d})_{A,-1}^{\dagger}(q \bar{q})_{A, 0}^{\dagger}-k_{2 \perp}^{-}(u \bar{d})_{A, 1}^{\dagger}(q \bar{q})_{A, 0}^{\dagger}\right] \\
& +\psi_{u \bar{d} q \bar{q}}^{(20)}(1,2,3,4)\left[k_{2 \perp}^{+}(u \bar{d})_{A,-1}^{\dagger}(q \bar{q})_{S, 0}^{\dagger}+k_{2 \perp}^{-}(u \bar{d})_{A, 1}^{\dagger}(q \bar{q})_{S, 0}^{\dagger}\right] \\
& +\psi_{u \bar{d} q \bar{q}}^{(21)}(1,2,3,4)\left[k_{3 \perp}^{+}(u \bar{d})_{A, 0}^{\dagger}(q \bar{q})_{A,-1}^{\dagger}-k_{3 \perp}^{-}(u \bar{d})_{A, 0}^{\dagger}(q \bar{q})_{A, 1}^{\dagger}\right] \\
& +\psi_{u \bar{d} q \bar{q}}^{(22)}(1,2,3,4)\left[k_{3 \perp}^{+}(u \bar{d})_{S, 0}^{\dagger}(q \bar{q})_{A,-1}^{\dagger}+k_{3 \perp}^{-}(u \bar{d})_{S, 0}^{\dagger}(q \bar{q})_{A, 1}^{\dagger}\right] \\
& +\psi_{u \bar{d} q \bar{q}}^{(23)}(1,2,3,4)\left[k_{3 \perp}^{+}(u \bar{d})_{A,-1}^{\dagger}(q \bar{q})_{A, 0}^{\dagger}-k_{3 \perp}^{-}(u \bar{d})_{A, 1}^{\dagger}(q \bar{q})_{A, 0}^{\dagger}\right] \\
& +\psi_{u \bar{d} q \bar{q}}^{(24)}(1,2,3,4)\left[k_{3 \perp}^{+}(u \bar{d})_{A,-1}^{\dagger}(q \bar{q})_{S, 0}^{\dagger}+k_{3 \perp}^{-}(u \bar{d})_{A, 1}^{\dagger}(q \bar{q})_{S, 0}^{\dagger}\right] \\
& +i \epsilon^{\alpha \beta} k_{2 \alpha} k_{3 \beta} \psi_{u \bar{d} q \bar{q}}^{(25)}(1,2,3,4)\left[k_{1 \perp}^{+}(u \bar{d})_{A, 0}^{\dagger}(q \bar{q})_{A,-1}^{\dagger}+k_{1 \perp}^{-}(u \bar{d})_{A, 0}^{\dagger}(q \bar{q})_{A, 1}^{\dagger}\right] \\
& +i \epsilon^{\alpha \beta} k_{2 \alpha} k_{3 \beta} \psi_{u \bar{u} q \bar{q}}^{(26)}(1,2,3,4)\left[k_{1 \perp}^{+}(u \bar{d})_{S, 0}^{\dagger}(q \bar{q})_{A,-1}^{\dagger}-k_{1 \perp}^{-}(u \bar{d})_{S, 0}^{\dagger}(q \bar{q})_{A, 1}^{\dagger}\right] \\
& +i \epsilon^{\alpha \beta} k_{2 \alpha} k_{3 \beta} \psi_{u \bar{u} q \bar{q}}^{(27)}(1,2,3,4)\left[k_{1 \perp}^{+}(u \bar{d})_{A,-1}^{\dagger}(q \bar{q})_{A, 0}^{\dagger}+k_{1 \perp}^{-}(u \bar{d})_{A, 1}^{\dagger}(q \bar{q})_{A, 0}^{\dagger}\right] \\
& +i \epsilon^{\alpha \beta} k_{2 \alpha} k_{3 \beta} \psi_{u \bar{d} q \bar{q}}^{(28)}(1,2,3,4)\left[k_{1 \perp}^{+}(u \bar{d})_{A,-1}^{\dagger}(q \bar{q})_{S, 0}^{\dagger}-k_{1 \perp}^{-}(u \bar{d})_{A, 1}^{\dagger}(q \bar{q})_{S, 0}^{\dagger}\right] \\
&
\end{aligned}
$$




$$
\begin{aligned}
& +i \epsilon^{\alpha \beta} k_{1 \alpha} k_{3 \beta} \psi_{u \bar{d} q \bar{q}}^{(29)}(1,2,3,4)\left[k_{2 \perp}^{+}(u \bar{d})_{A, 0}^{\dagger}(q \bar{q})_{A,-1}^{\dagger}+k_{2 \perp}^{-}(u \bar{d})_{A, 0}^{\dagger}(q \bar{q})_{A, 1}^{\dagger}\right] \\
& +i \epsilon^{\alpha \beta} k_{1 \alpha} k_{3 \beta} \psi_{u \bar{d} q \bar{q}}^{(30)}(1,2,3,4)\left[k_{2 \perp}^{+}(u \bar{d})_{S, 0}^{\dagger}(q \bar{q})_{A,-1}^{\dagger}-k_{2 \perp}^{-}(u \bar{d})_{S, 0}^{\dagger}(q \bar{q})_{A, 1}^{\dagger}\right] \\
& +i \epsilon^{\alpha \beta} k_{1 \alpha} k_{3 \beta} \psi_{u \bar{d} q \bar{q}}^{(31)}(1,2,3,4)\left[k_{2 \perp}^{+}(u \bar{d})_{A,-1}^{\dagger}(q \bar{q})_{A, 0}^{\dagger}+k_{2 \perp}^{-}(u \bar{d})_{A, 1}^{\dagger}(q \bar{q})_{A, 0}^{\dagger}\right] \\
& +i \epsilon^{\alpha \beta} k_{1 \alpha} k_{3 \beta} \psi_{u \bar{d} q \bar{q}}^{(32)}(1,2,3,4)\left[k_{2 \perp}^{+}(u \bar{d})_{A,-1}^{\dagger}(q \bar{q})_{S, 0}^{\dagger}-k_{2 \perp}^{-}(u \bar{d})_{A, 1}^{\dagger}(q \bar{q})_{S, 0}^{\dagger}\right] \\
& +i \epsilon^{\alpha \beta} k_{1 \alpha} k_{2 \beta} \psi_{u \bar{d} q \bar{q}}^{(33)}(1,2,3,4)\left[k_{3 \perp}^{+}(u \bar{d})_{A, 0}^{\dagger}(q \bar{q})_{A,-1}^{\dagger}+k_{3 \perp}^{-}(u \bar{d})_{A, 0}^{\dagger}(q \bar{q})_{A, 1}^{\dagger}\right] \\
& +i \epsilon^{\alpha \beta} k_{1 \alpha} k_{2 \beta} \psi_{u \bar{d} q \bar{q}}^{(34)}(1,2,3,4)\left[k_{3 \perp}^{+}(u \bar{d})_{S, 0}^{\dagger}(q \bar{q})_{A,-1}^{\dagger}-k_{3 \perp}^{-}(u \bar{d})_{S, 0}^{\dagger}(q \bar{q})_{A, 1}^{\dagger}\right] \\
& +i \epsilon^{\alpha \beta} k_{1 \alpha} k_{2 \beta} \psi_{u \bar{d} q \bar{q}}^{(35)}(1,2,3,4)\left[k_{3 \perp}^{+}(u \bar{d})_{A,-1}^{\dagger}(q \bar{q})_{A, 0}^{\dagger}+k_{3 \perp}^{-}(u \bar{d})_{A, 1}^{\dagger}(q \bar{q})_{A, 0}^{\dagger}\right] \\
& \left.+i \epsilon^{\alpha \beta} k_{1 \alpha} k_{2 \beta} \psi_{u \bar{d} q \bar{q}}^{(36)}(1,2,3,4)\left[k_{3 \perp}^{+}(u \bar{d})_{A,-1}^{\dagger}(q \bar{q})_{S, 0}^{\dagger}-k_{3 \perp}^{-}(u \bar{d})_{A, 1}^{\dagger}(q \bar{q})_{S, 0}^{\dagger}\right]\right\}|0\rangle .
\end{aligned}
$$

For $\left|l_{z}\right|=2$, the quark helicity must be $\lambda_{u \bar{d}}= \pm 1$ and $\lambda_{u \bar{u}}= \pm 1$. We have the following 9 independent amplitudes,

$$
\begin{aligned}
\left|\pi^{+}\right\rangle_{u \bar{d} q \bar{q}}^{\mid l_{z}=2}= & \int d[1] d[2] d[3] d[4] \frac{\delta_{i j} \delta_{k l}}{3}\{ \\
& \psi_{u \bar{d} q \bar{q}}^{(37)}(1,2,3,4)\left[k_{1 \perp}^{+} k_{1 \perp}^{+}(u \bar{u})_{A,-1}^{\dagger}(q \bar{q})_{A,-1}^{\dagger}-k_{1 \perp}^{-} k_{1 \perp}^{-}(u \bar{d})_{A, 1}^{\dagger}(q \bar{q})_{A, 1}^{\dagger}\right] \\
& +\psi_{u \bar{d} q \bar{q}}^{(38)}(1,2,3,4)\left[k_{2 \perp}^{+} k_{2 \perp}^{+}(u \bar{d})_{A,-1}^{\dagger}(q \bar{q})_{A,-1}^{\dagger}-k_{2 \perp}^{-} k_{2 \perp}^{-}(u \bar{d})_{A, 1}^{\dagger}(q \bar{q})_{A, 1}^{\dagger}\right] \\
& +\psi_{u \bar{d} q \bar{q}}^{(39)}(1,2,3,4)\left[k_{3 \perp}^{+} k_{3 \perp}^{+}(u \bar{d})_{A,-1}^{\dagger}(q \bar{q})_{A,-1}^{\dagger}-k_{3 \perp}^{-} k_{3 \perp}^{-}(u \bar{d})_{A, 1}^{\dagger}(q \bar{q})_{A, 1}^{\dagger}\right] \\
& +\psi_{u \bar{d} q \bar{q}}^{(40)}(1,2,3,4)\left[k_{1 \perp}^{+} k_{2 \perp}^{+}(u \bar{d})_{A,-1}^{\dagger}(q \bar{q})_{A,-1}^{\dagger}-k_{1 \perp}^{-} k_{2 \perp}^{-}(u \bar{d})_{A, 1}^{\dagger}(q \bar{q})_{A, 1}^{\dagger}\right] \\
& +\psi_{u \bar{d} q \bar{q} \bar{q}}^{(41)}(1,2,3,4)\left[k_{1 \perp}^{+} k_{3 \perp}^{+}(u \bar{d})_{A,-1}^{\dagger}(q \bar{q})_{A,-1}^{\dagger}-k_{1 \perp}^{-} k_{3 \perp}^{-}(u \bar{d})_{A, 1}^{\dagger}(q \bar{q})_{A, 1}^{\dagger}\right] \\
& +\psi_{u \bar{d} q \bar{q}}^{(42)}(1,2,3,4)\left[k_{2 \perp}^{+} k_{3 \perp}^{+}(u \bar{d})_{A,-1}^{\dagger}(q \bar{q})_{A,-1}^{\dagger}-k_{2 \perp}^{-} k_{3 \perp}^{-}(u \bar{d})_{A, 1}^{\dagger}(q \bar{q})_{A, 1}^{\dagger}\right] \\
& +i \epsilon^{\alpha \beta} k_{2 \alpha} k_{3 \beta} \psi_{u \bar{d} q \bar{q}}^{(43)}(1,2,3,4)\left[k_{1 \perp}^{+} k_{1 \perp}^{+}(u \bar{d})_{A,-1}^{\dagger}(q \bar{q})_{A,-1}^{\dagger}+k_{1 \perp}^{-} k_{1 \perp}^{-}(u \bar{d})_{A, 1}^{\dagger}(q \bar{q})_{A, 1}^{\dagger}\right] \\
& +i \epsilon^{\alpha \beta} k_{1 \alpha} k_{3 \beta} \psi_{u \bar{d} \bar{q} \bar{q}}^{(44)}(1,2,3,4)\left[k_{2 \perp}^{+} k_{2 \perp}^{+}(u \bar{d})_{A,-1}^{\dagger}(q \bar{q})_{A,-1}^{\dagger}+k_{2 \perp}^{-} k_{2 \perp}^{-}(u \bar{d})_{A, 1}^{\dagger}(q \bar{q})_{A, 1}^{\dagger}\right] \\
& \left.+i \epsilon^{\alpha \beta} k_{1 \alpha} k_{2 \beta} \psi_{u \bar{q} \bar{q}}^{(45)}(1,2,3,4)\left[k_{3 \perp}^{+} k_{3 \perp}^{+}(u \bar{d})_{A,-1}^{\dagger}(q \bar{q})_{A,-1}^{\dagger}+k_{3 \perp}^{-} k_{3 \perp}^{-}(u \bar{d})_{A, 1}^{\dagger}(q \bar{q})_{A, 1}^{\dagger}\right]\right\}|0\rangle .
\end{aligned}
$$

In summary, we have found 45 independent amplitudes. Similarly, we have another 45 amplitudes for the color structure $\frac{1}{3} \delta_{i l} \delta_{j k}$. Together, we have 90 independent amplitudes for the $u \bar{d} q \bar{q}$ component.

The above formalism can also be used to construct the amplitudes for the $u \bar{d} s \bar{s}$ and $u \bar{d} c \bar{c}$ components in $\pi^{+}$. The total number of independent amplitudes are 90 in both cases. These amplitudes can be used to describe the intrinsic strange and/or charm contributions to the hadronic processes involving $\pi$, e.g., $J / \psi \rightarrow \rho \pi$ decays [26]. 


\section{WAVE-FUNCTION AMPLITUDES FOR THE $\rho^{+}$-MESON}

The method in the last section can be straightforwardly used to construct the lightcone wave-function amplitudes for the $\rho$ mesons. Strictly speaking, the $\rho$ meson is not an eigenstate of the QCD hamiltonian, it appears as resonances in, for example, $\pi \pi$ scattering. However, we regard in the following discussion the $\rho$ meson as if a bound state of quarks and gluons. The relevant studies of the distribution amplitudes for $\rho$ mesons can be found in [27, 28].

Because $\rho$ is a vector meson, it has three helicity states, i.e., $\Lambda=0, \pm 1$, corresponding to longitudinal $(\Lambda=0)$ and transverse $(\Lambda= \pm 1)$ polarizations. The wave functions for the $\Lambda=0$ state can be obtained, in principle, from those of the $\Lambda= \pm 1$ states by using angular momentum raising and lowering operators. In practice, however, these operators involve complicated quark-gluon interactions in light-cone quantization, and the constraint becomes a very complicated equation involving all higher Fock states. Since we are interested in the components of few partons, we may regard the different helicity states as quasiindependent. Nonetheless, the $\Lambda=-1$ state can be obtained from $\Lambda=+1$ state using parity transformation.

The wave-function amplitudes for the helicity $\Lambda=0$ state can be easily obtained from

those in the last section, taking into account the difference on $\hat{Y}$ transformation property between $\pi$ and $\rho$, i.e.,

$$
\hat{Y}\left|\rho^{+}, \Lambda=0\right\rangle=\left|\rho^{+}, \Lambda=0\right\rangle,
$$

compared to Eq. (12). Hence the Fock expansion listed in the last section can be transformed to that of $\left|\rho^{+}, 0\right\rangle$, except some signs must be changed. In particular, the total number of the independent amplitudes will be the same.

For example, the two quark component for $\left|\rho^{+}, 0\right\rangle$ has 2 independent amplitudes

$$
\begin{aligned}
\left|\rho^{+}, 0\right\rangle_{u \bar{d}}^{l_{z}=0} & =\int d[1] d[2] \psi_{u \bar{d}}^{(1)}(1,2) \frac{1}{\sqrt{3}}\left[u_{\uparrow i}^{\dagger}(1) \bar{d}_{\downarrow i}^{\dagger}(2)+u_{\downarrow i}^{\dagger}(1) \bar{d}_{\uparrow i}^{\dagger}(2)\right]|0\rangle \\
\left|\rho^{+}, 0\right\rangle_{u \bar{d}}^{\left|l_{z}\right|=1} & =\int d[1] d[2] \psi_{u \bar{d}}^{(2)}(1,2) \frac{1}{\sqrt{3}}\left[k_{1 \perp}^{-} u_{\uparrow i}^{\dagger}(1) \bar{d}_{\uparrow i}^{\dagger}(2)-k_{1 \perp}^{+} u_{\downarrow i}^{\dagger}(1) \bar{d}_{\downarrow i}^{\dagger}(2)\right]|0\rangle .
\end{aligned}
$$

Here we have used the same notation for the amplitudes, assuming no confusion will arise. For the $u \bar{d} g$ component, we have

$$
\begin{aligned}
\left|\rho^{+}, 0\right\rangle_{u \bar{d} g}^{l_{z}=0}= & \int d[1] d[2] d[3] \frac{T_{i j}^{a}}{2}\left\{\psi_{u \bar{d} g}^{(1)}(1,2,3)\left[(u \bar{d})_{A, 1}^{\dagger} g_{\downarrow}^{a \dagger}(3)+(u \bar{d})_{A,-1}^{\dagger} g_{\uparrow}^{a \dagger}(3)\right]|0\rangle\right. \\
& \left.+i \epsilon^{\alpha \beta} k_{1 \alpha} k_{2 \beta} \psi_{u \bar{d} g}^{(2)}(1,2,3)\left[(u \bar{d})_{A, 1}^{\dagger} g_{\downarrow}^{a \dagger}(3)-(u \bar{d})_{A,-1}^{\dagger} g_{\uparrow}^{a \dagger}(3)\right]\right\}|0\rangle, \\
\left|\rho^{+}, 0\right\rangle_{u \bar{d} g}^{l_{z} \mid=1}= & \int d[1] d[2] d[3] \frac{T_{i j}^{a}}{2}\left\{\psi_{u \bar{d} g}^{(3)}(1,2,3)\left[k_{1 \perp}^{+}(u \bar{d})_{A, 0}^{\dagger} g_{\downarrow}^{a \dagger}(3)+k_{1 \perp}^{-}(u \bar{d})_{A, 0}^{\dagger} g_{\uparrow}^{a \dagger}(3)\right]\right. \\
& +\psi_{u \bar{d} g}^{(4)}(1,2,3)\left[k_{2 \perp}^{+}(u \bar{d})_{A, 0}^{\dagger} g_{\downarrow}^{a \dagger}(3)+k_{2 \perp}^{-}(u \bar{d})_{A, 0}^{\dagger} g_{\uparrow}^{a \dagger}(3)\right] \\
& +\psi_{u \bar{d} g}^{(5)}(1,2,3)\left[k_{1 \perp}^{+}(u \bar{d})_{S, 0}^{\dagger} g_{\downarrow}^{a \dagger}(3)-k_{1 \perp}^{-}(u \bar{d})_{S, 0}^{\dagger} g_{\uparrow}^{a \dagger}(3)\right] \\
& \left.+\psi_{u \bar{d} g}^{(6)}(1,2,3)\left[k_{2 \perp}^{+}(u \bar{d})_{S, 0}^{\dagger} g_{\downarrow}^{a \dagger}(3)-k_{2 \perp}^{-}(u \bar{d})_{S, 0}^{\dagger} g_{\uparrow}^{a \dagger}(3)\right]\right\}|0\rangle,
\end{aligned}
$$




$$
\begin{aligned}
\left|\rho^{+}, 0\right\rangle_{u \bar{d} g}^{\left|l_{z}\right|=2}= & \int d[1] d[2] d[3] \frac{T_{i j}^{a}}{2}\left\{\psi_{u \bar{d} g}^{(7)}(1,2,3)\left[k_{1 \perp}^{+} k_{1 \perp}^{+}(u \bar{d})_{A,-1}^{\dagger} g_{\downarrow}^{a \dagger}(3)+k_{1 \perp}^{-} k_{1 \perp}^{-}(u \bar{d})_{A, 1}^{\dagger} g_{\uparrow}^{a \dagger}(3)\right]\right. \\
& +\psi_{u \bar{d} g}^{(8)}(1,2,3)\left[k_{1 \perp}^{+} k_{2 \perp}^{+}(u \bar{d})_{A,-1}^{\dagger} g_{\downarrow}^{a \dagger}(3)+k_{1 \perp}^{-} k_{2 \perp}^{-}(u \bar{d})_{A, 1}^{\dagger} g_{\uparrow}^{a \dagger}(3)\right] \\
& \left.+\psi_{u \bar{d} g}^{(9)}(1,2,3)\left[k_{2 \perp}^{+} k_{2 \perp}^{+}(u \bar{d})_{A,-1}^{\dagger} g_{\downarrow}^{a \dagger}(3)+k_{2 \perp}^{-} k_{2 \perp}^{-}(u \bar{d})_{A, 1}^{\dagger} g_{\uparrow}^{a \dagger}(3)\right]\right\}|0\rangle .
\end{aligned}
$$

We will not repeat the cases for four partons.

The helicity $\Lambda=1 \rho$-meson state, $\left|\rho^{+}, 1\right\rangle$, can be constructed similarly. For example, the $u \bar{d}$ component defined 4 independent amplitudes, corresponding to $l_{z}=0,1,2$,

$$
\begin{aligned}
\left|\rho^{+}, 1\right\rangle_{u \bar{d}}^{l_{z}=0}= & \int d[1] d[2] \psi_{u \bar{d}}^{(1)}(1,2) \frac{1}{\sqrt{3}}\left[u_{\uparrow i}^{\dagger}(1) \bar{d}_{\uparrow i}^{\dagger}(2)\right]|0\rangle \\
\left|\rho^{+}, 1\right\rangle_{u \bar{d}}^{l_{z}=1}= & \int d[1] d[2]\left\{k_{1 \perp}^{+} \psi_{u \bar{d}}^{(2)}(1,2) \frac{1}{\sqrt{3}}\left[u_{\uparrow i}^{\dagger}(1) \bar{d}_{\downarrow i}^{\dagger}(2)+u_{\downarrow i}^{\dagger}(1) \bar{d}_{\uparrow i}^{\dagger}(2)\right]\right. \\
& \left.+k_{1 \perp}^{+} \psi_{u \bar{d}}^{(3)}(1,2) \frac{1}{\sqrt{3}}\left[u_{\uparrow i}^{\dagger}(1) \bar{d}_{\downarrow i}^{\dagger}(2)-u_{\downarrow i}^{\dagger}(1) \bar{d}_{\uparrow i}^{\dagger}(2)\right]\right\}|0\rangle \\
\left|\rho^{+}, 1\right\rangle_{u \bar{d}}^{l_{z}=2}= & \int d[1] d[2]\left(k_{1 \perp}^{+}\right)^{2} \psi_{u \bar{d}}^{(4)}(1,2) \frac{1}{\sqrt{3}}\left[u_{\downarrow i}^{\dagger}(1) \bar{d}_{\downarrow i}^{\dagger}(2)\right]|0\rangle .
\end{aligned}
$$

Here again we use the same notation for the wave-function amplitudes although they can be very different from those in the $\Lambda=0$ state. In fact, even the number of independent amplitudes for a given number of partons is different.

For the $u \bar{d} g$ component, the parton orbital angular momentum can be $l_{z}=-1,0,1,2$, and 3. For $l_{z}=0$, we find 4 independent amplitudes

$$
\begin{aligned}
\left|\rho^{+}, 1\right\rangle_{u \bar{d} g}^{l_{z}=0}= & \int d[1] d[2] d[3] \frac{T_{i j}^{a}}{2}\left\{\left(\psi_{u \bar{d} g}^{(1)}(1,2,3)+i \epsilon^{\alpha \beta} k_{1 \alpha} k_{2 \beta} \psi_{u \bar{d} g}^{(2)}(1,2,3)\right)\left[(u \bar{d})_{A, 0}^{\dagger} g_{\uparrow}^{a \dagger}(3)\right]\right. \\
& \left.+\left(\psi_{u \bar{d} g}^{(3)}(1,2,3)+i \epsilon^{\alpha \beta} k_{1 \alpha} k_{2 \beta} \psi_{u \bar{d} g}^{(4)}(1,2,3)\right)\left[(u \bar{d})_{S, 0}^{\dagger} g_{\uparrow}^{a \dagger}(3)\right]\right\}|0\rangle .
\end{aligned}
$$

For $l_{z}=-1$, we have 2 independent amplitudes

$$
\begin{aligned}
\left|\rho^{+}, 1\right\rangle_{u \bar{d} g}^{l_{z}=-1}= & \int d[1] d[2] d[3] \frac{T_{i j}^{a}}{2}\left\{\psi_{u \bar{d} g}^{(5)}(1,2,3)\left[k_{1 \perp}^{-}(u \bar{d})_{A, 1}^{\dagger} g_{\uparrow}^{a \dagger}(3)\right]\right. \\
& \left.+\psi_{u \bar{d} g}^{(6)}(1,2,3)\left[k_{2 \perp}^{-}(u \bar{d})_{A, 1}^{\dagger} g_{\uparrow}^{a \dagger}(3)\right]\right\}|0\rangle .
\end{aligned}
$$

For $l_{z}=1$, we have 4 independent amplitudes

$$
\begin{aligned}
\left|\rho^{+}, 1\right\rangle_{u \bar{d} g}^{l_{z}=1}= & \int d[1] d[2] d[3] \frac{T_{i j}^{a}}{2}\left\{k_{1 \perp}^{+} \psi_{u \bar{d} g}^{(7)}(1,2,3)\left[(u \bar{d})_{A, 1}^{\dagger} g_{\downarrow}^{a \dagger}(3)+(u \bar{d})_{A,-1}^{\dagger} g_{\uparrow}^{a \dagger}(3)\right]\right. \\
& +k_{2 \perp}^{+} \psi_{u \bar{d} g}^{(8)}(1,2,3)\left[(u \bar{d})_{A, 1}^{\dagger} g_{\downarrow}^{a \dagger}(3)+(u \bar{d})_{A,-1}^{\dagger} g_{\uparrow}^{a \dagger}(3)\right] \\
& +k_{1 \perp}^{+} \psi_{u \bar{d} g}^{(9)}(1,2,3)\left[(u \bar{d})_{A, 1}^{\dagger} g_{\downarrow}^{a \dagger}(3)-(u \bar{d})_{A,-1}^{\dagger} g_{\uparrow}^{a \dagger}(3)\right] \\
& \left.+k_{2 \perp}^{+} \psi_{u \bar{d} g}^{(10)}(1,2,3)\left[(u \bar{d})_{A, 1}^{\dagger} g_{\downarrow}^{a \dagger}(3)-(u \bar{d})_{A,-1}^{\dagger} g_{\uparrow}^{a \dagger}(3)\right]\right\}|0\rangle .
\end{aligned}
$$


For $l_{z}=2$, we have 6 independent amplitudes

$$
\begin{aligned}
\left|\rho^{+}, 1\right\rangle_{u \bar{d} g}^{l_{z}=2}= & \int d[1] d[2] d[3] \frac{T_{i j}^{a}}{2}\left\{k_{1 \perp}^{+} k_{1 \perp}^{+} \psi_{u \bar{d} g}^{(11)}(1,2,3)\left[(u \bar{d})_{A, 0}^{\dagger} g_{\downarrow}^{a \dagger}(3)\right]\right. \\
& +k_{1 \perp}^{+} k_{2 \perp}^{+} \psi_{u \bar{d} g}^{(12)}(1,2,3)\left[(u \bar{d})_{A, 0}^{\dagger} g_{\downarrow}^{a \dagger}(3)\right] \\
& +k_{2 \perp}^{+} k_{2 \perp}^{+} \psi_{u \bar{d} g}^{(13)}(1,2,3)\left[(u \bar{d})_{A, 0}^{\dagger} g_{\downarrow}^{a \dagger}(3)\right] \\
& +k_{1 \perp}^{+} k_{1 \perp}^{+} \psi_{u \bar{d} g}^{(14)}(1,2,3)\left[(u \bar{d})_{S, 0}^{\dagger} g_{\downarrow}^{a \dagger}(3)\right] \\
& +k_{1 \perp}^{+} k_{2 \perp}^{+} \psi_{u \bar{d} g}^{(15)}(1,2,3)\left[(u \bar{d})_{S, 0}^{\dagger} g_{\downarrow}^{a \dagger}(3)\right] \\
& \left.+k_{2 \perp}^{+} k_{2 \perp}^{+} \psi_{u \bar{d} g}^{(16)}(1,2,3)\left[(u \bar{d})_{S, 0}^{\dagger} g_{\downarrow}^{a \dagger}(3)\right]\right\}|0\rangle .
\end{aligned}
$$

For $l_{z}=3$, we have 4 independent amplitudes,

$$
\begin{aligned}
\left|\rho^{+}, 1\right\rangle_{u \bar{d} g}^{l_{z}=3}= & \int d[1] d[2] d[3] \frac{T_{i j}^{a}}{2}\left\{\left(k_{1 \perp}^{+}\right)^{3} \psi_{u \bar{d} g}^{(17)}(1,2,3)\left[(u \bar{d})_{A,-1}^{\dagger} g_{\downarrow}^{a \dagger}(3)\right]\right. \\
& +\left(k_{2 \perp}^{+}\right)^{3} \psi_{u \bar{d} g}^{(18)}(1,2,3)\left[(u \bar{d})_{A,-1}^{\dagger} g_{\downarrow}^{a \dagger}(3)\right] \\
& +\left(k_{1 \perp}^{+}\right)^{2} k_{2 \perp}^{+} \psi_{u \bar{d} g}^{(19)}(1,2,3)\left[(u \bar{d})_{A,-1}^{\dagger} g_{\downarrow}^{a \dagger}(3)\right] \\
& \left.+k_{1 \perp}^{+}\left(k_{2 \perp}^{+}\right)^{2} \psi_{u \bar{d} g}^{(20)}(1,2,3)\left[(u \bar{d})_{A,-1}^{\dagger} g_{\downarrow}^{a \dagger}(3)\right]\right\}|0\rangle .
\end{aligned}
$$

In total, we have 20 amplitudes, compared with the $\Lambda=0$ case where we have only 9 . For simplicity, we will not consider those amplitudes with four partons.

\section{WAVE-FUNCTION AMPLITUDES FOR THE NUCLEON}

In this section, we enumerate the number of independent amplitudes for the nucleon, and more specifically for the proton. For the neutron, one just interchange the up and down quarks assuming isospin symmetry. Our expansion is also valid for the whole baryon octet, except the flavor structure need to be modified accordingly.

We consider only the state with positive helicity. The negative helicity state can be obtained simply from the modified parity transformation $\hat{Y}$. Three quark amplitudes have been studied extensively in Ref. [5]. The new result here includes three quark plus one gluon amplitudes. One can add an additional pair of sea quarks into the valence component, but the result is very complicated and we will not show it here.

\section{A. The uud Component}

The quark distribution amplitudes describing the three-quark component of the proton have been studied extensively in the literature [4, 14, 27, 29, 30, 31]. The wave-function

amplitudes keeping full partons transverse-momentum dependence have been studied in 
Ref. [5]. From the approach advocated here, we immediate have for $l_{z}=0$ and $l_{z}=1$,

$$
\begin{aligned}
|P \uparrow\rangle_{u u d}^{l_{z}=0}= & \int d[1] d[2] d[3]\left(\psi_{u u d}^{(1)}(1,2,3)+i \epsilon^{\alpha \beta} k_{1 \alpha} k_{2 \beta} \psi_{u u d}^{(2)}(1,2,3)\right) \\
& \times \frac{\epsilon^{i j k}}{\sqrt{6}} u_{i \uparrow}^{\dagger}(1)\left(u_{j \downarrow}^{\dagger}(2) d_{k \uparrow}^{\dagger}(3)-d_{j \downarrow}^{\dagger}(2) u_{k \uparrow}^{\dagger}(3)\right)|0\rangle, \\
|P \uparrow\rangle_{u u d}^{l_{z}=1}= & \int d[1] d[2] d[3]\left(k_{1 \downarrow}^{+} \psi_{u u d}^{(3)}(1,2,3)+k_{2 \perp}^{+} \psi_{u u d}^{(4)}(1,2,3)\right) \\
& \times \frac{\epsilon^{i j k}}{\sqrt{6}}\left(u_{i \uparrow}^{\dagger}(1) u_{j \downarrow}^{\dagger}(2) d_{k \downarrow}^{\dagger}(3)-d_{i \uparrow}^{\dagger}(1) u_{j \downarrow}^{\dagger}(2) u_{k \downarrow}^{\dagger}(3)\right)|0\rangle .
\end{aligned}
$$

For $l_{z}=-1$, we have

$$
\begin{aligned}
|P \uparrow\rangle_{u u d}^{l_{z}=-1}= & \int d[1] d[2] d[3] k_{2 \perp}^{-} \psi_{u u d}^{(5)}(1,2,3) \\
& \times \frac{\epsilon^{i j k}}{\sqrt{6}} u_{i \uparrow}^{\dagger}(1)\left(u_{j \uparrow}^{\dagger}(2) d_{k \uparrow}^{\dagger}(3)-d_{j \uparrow}^{\dagger}(2) u_{k \uparrow}^{\dagger}(3)\right)|0\rangle,
\end{aligned}
$$

where we have used quark 2 and 3 anti-symmetry. Likewise, we have,

$$
\begin{aligned}
|P \uparrow\rangle_{u u d}^{l_{z}=2}= & \int d[1] d[2] d[3] k_{1 \perp}^{+} k_{3 \perp}^{+} \psi_{u u d}^{(6)}(1,2,3) \\
& \times \frac{\epsilon^{i j k}}{\sqrt{6}} u_{\downarrow \downarrow}^{\dagger}(1)\left(d_{j \downarrow}^{\dagger}(2) u_{k \downarrow}^{\dagger}(3)-u_{j \downarrow}^{\dagger}(2) d_{k \downarrow}^{\dagger}(3)\right)|0\rangle .
\end{aligned}
$$

where, in principle, there is an additional term $k_{2 \perp}^{+} k_{3 \perp}^{+} \psi_{u u d}^{\left(6^{\prime}\right)}(1,2,3)$. However, after using 2 and 3 anti-symmetry and 1 and 2 symmetry, it can shown that this term can be reduced to the term shown above.

\section{B. The $u u d+g$ Component}

Let us first consider the isospin symmetry. With three quarks uud, one can construct two possible $I=1 / 2$ isospin combinations:

$$
\begin{aligned}
& u(u d-d u), \\
& 2 d u u-u d u-u u d .
\end{aligned}
$$

However, the second flavor structure can be reduced to the first one after some shuffling of the particle labels. Therefore, we shall only consider the first structure for the flavor wave function, taking into account all possible color and spin assignments for the three quarks and one gluon.

Since the gluon belongs to color-octet, the three quarks must couple to a color-octet. For the three quarks, $u_{i}, u_{j}, d_{k}$, there are two possible ways to couple to color-octet

$$
3 \times 3 \times 3=(6+\overline{3}) \times 3=1+8+8+10 .
$$

We can have the first two quarks couple to $\overline{3}$, and then couple them to the third quark to form

a color-octet. In this case, we have an overall color factor, $\epsilon^{i j l} T_{l k}^{a}$. Similarly, we can also have 
other two color factors, $\epsilon^{j k l} T_{l i}^{a}$ and $\epsilon^{k i l} T_{l j}^{a}$. However, the above three are not independent, because $\epsilon^{i j l} T_{l k}^{a}+\epsilon^{j k l} T_{l i}^{a}+\epsilon^{k i l} T_{l j}^{a}=0$. If we use the isospin structure $u_{i}\left(u_{j} d_{k}-d_{j} u_{k}\right)$, the best way to select the two independent color structures is to have the indices of $j k$ to be antisymmetric or symmetric:

$$
\epsilon^{j k l} T_{l i}^{a}, \quad \epsilon^{i j l} T_{l k}^{a}+\epsilon^{i k l} T_{l j}^{a} .
$$

For the Fock component of $u u d g$, the total quark helicity can be $\lambda_{u u d}=-3 / 2$, $1 / 2,1 / 2,3 / 2$, and the gluon helicity $\lambda_{g}= \pm 1$. The parton orbital angular momentum projection can have the following values, $l_{z}=0,1,2,3,-1,-2$.

For $l_{z}=0$, the parton helicity can either be $\lambda_{\text {uud }}=3 / 2$ and $\lambda_{g}=-1$, or $\lambda_{\text {uud }}=-1 / 2$ and $\lambda_{g}=1$. For the first case, because the total quark helicity $\lambda_{u u d}=3 / 2$, the three quarks are all in helicity-1/2 states, and we only have one spin structure, i.e., $u_{i \uparrow}\left(u_{j \uparrow} d_{k \uparrow}-d_{j \uparrow} u_{k \uparrow}\right)$. Therefore, we can write down 3 independent amplitudes,

$$
\begin{aligned}
|P \uparrow\rangle_{u u d g}^{l_{z}=0}= & \int d[1] d[2] d[3] d[4] \\
& \left(\psi_{u u d g}^{(1)}(1,2,3,4)+i \epsilon^{\alpha \beta} k_{1 \alpha} k_{2 \beta} \psi_{u u d g}^{(2)}(1,2,3,4)+i \epsilon^{\alpha \beta} k_{2 \alpha} k_{3 \beta} \psi_{u u d g}^{(3)}(1,2,3,4)\right) \\
& \times \frac{\epsilon^{j k l} T_{l i}^{a}}{2}\left[u_{i \uparrow}^{\dagger}(1)\left(u_{j \uparrow}^{\dagger}(2) d_{k \uparrow}^{\dagger}(3)-d_{j \uparrow}^{\dagger}(2) u_{k \uparrow}^{\dagger}(3)\right) g_{\downarrow}^{a \dagger}(4)\right]|0\rangle
\end{aligned}
$$

Here, we have used the $2 \leftrightarrow 3$ symmetry to reduce the number of the independent amplitudes. For example, $i \epsilon^{\alpha \beta} k_{1 \alpha} k_{3 \beta}$ term can be obtained from $i \epsilon^{\alpha \beta} k_{1 \alpha} k_{2 \beta}$ by 2 and 3 exchange, and hence the former is not independent. We have the following (anti)symmetric properties for some amplitudes, $\psi_{\text {uudg }}^{(1)}(1,2,3,4)=-\psi_{\text {uudg }}^{(1)}(1,3,2,4)$ and $\psi_{\text {uudg }}^{(3)}(1,2,3,4)=\psi_{\text {uudg }}^{(3)}(1,3,2,4)$.

In the second case, the total quark helicity $\lambda_{\text {uud }}=-1 / 2$. There are three possible spin structures for the three quarks,

$$
\begin{aligned}
& u_{i \downarrow}(1)\left(u_{j \downarrow}(2) d_{k \uparrow}(3)-d_{j \downarrow}(2) u_{k \uparrow}(3)\right), \\
& u_{i \downarrow}(1)\left(u_{j \uparrow}(2) d_{k \downarrow}(3)-d_{j \uparrow}(2) u_{k \downarrow}(3)\right), \\
& u_{i \uparrow}(1)\left(u_{j \downarrow}(2) d_{k \downarrow}(3)-d_{j \downarrow}(2) u_{k \downarrow}(3)\right) .
\end{aligned}
$$

However, the associated color structures indicate that there is a (anti)symmetric relation between the two indices $j$ and $k$. Thus, the first two spin structures are equivalent to each other under 2 and 3 exchange. In the following, we will only keep one of the first two spin structures. The above observation also applies to the total quark helicity $\lambda_{u u d}=1 / 2$ case. Taking into account this, we find 7 independent amplitudes

$$
\begin{aligned}
|P \uparrow\rangle_{u u d g}^{l_{z}=0}= & \int d[1] d[2] d[3] d[4]\left\{\left(\psi_{u u d g}^{(4)}(1,2,3,4)+i \epsilon^{\alpha \beta} k_{1 \alpha} k_{2 \beta} \psi_{u u d g}^{(5)}(1,2,3,4)\right.\right. \\
& \left.+i \epsilon^{\alpha \beta} k_{1 \alpha} k_{3 \beta} \psi_{u u d g}^{(6)}(1,2,3,4)+i \epsilon^{\alpha \beta} k_{2 \alpha} k_{3 \beta} \psi_{u u d g}^{(7)}(1,2,3,4)\right) \\
& \times \frac{\epsilon^{j k l} T_{l i}^{a}}{2}\left[u_{i \downarrow}^{\dagger}(1)\left(u_{j \uparrow}^{\dagger}(2) d_{k \downarrow}^{\dagger}(3)-d_{j \uparrow}^{\dagger}(2) u_{k \downarrow}^{\dagger}(3)\right) g_{\uparrow}^{a \dagger}(4)\right] \\
& +\left(\psi_{u u d g}^{(8)}(1,2,3,4)+i \epsilon^{\alpha \beta} k_{1 \alpha} k_{2 \beta} \psi_{u u d g}^{(9)}(1,2,3,4)+i \epsilon^{\alpha \beta} k_{2 \alpha} k_{3 \beta} \psi_{u u d g}^{(10)}(1,2,3,4)\right) \\
& \left.\times \frac{\epsilon^{j k l} T_{l i}^{a}}{2}\left[u_{i \uparrow}^{\dagger}(1)\left(u_{j \downarrow}^{\dagger}(2) d_{k \downarrow}^{\dagger}(3)-d_{j \downarrow}^{\dagger}(2) u_{k \downarrow}^{\dagger}(3)\right) g_{\uparrow}^{a \dagger}(4)\right]\right\}|0\rangle .
\end{aligned}
$$


Again, the 2 and 3 symmetry in the above equation has been used to reduce the number of independent amplitudes. Moreover, it implies the relations $\psi_{\text {uudg }}^{(8)}(1,2,3,4)=-\psi_{\text {uudg }}^{(8)}(1,3,2,4)$ and $\psi_{\text {uudg }}^{(10)}(1,2,3,4)=\psi_{\text {uudg }}^{(10)}(1,3,2,4)$.

For $l_{z}=1$, the parton helicity can either be $\lambda_{u u d}=1 / 2$ and $\lambda_{g}=-1$, or $\lambda_{\text {uud }}=-3 / 2$ and $\lambda_{g}=1$. In the first case, we define 10 independent amplitudes

$$
\begin{aligned}
|P \uparrow\rangle_{u u d g}^{l_{z}=1}= & \int d[1] d[2] d[3] d[4]\left\{\left(k_{1 \perp}^{+}\left(\psi_{u u d g}^{(11)}(1,2,3,4)+i \epsilon^{\alpha \beta} k_{2 \alpha} k_{3 \beta} \psi_{u u d g}^{(12)}(1,2,3,4)\right)\right.\right. \\
& +k_{2 \perp}^{+}\left(\psi_{u u d g}^{(13)}(1,2,3,4)+i \epsilon^{\alpha \beta} k_{1 \alpha} k_{3 \beta} \psi_{u u d g}^{(14)}(1,2,3,4)\right) \\
& \left.+k_{3 \perp}^{+}\left(\psi_{u u d g}^{(15)}(1,2,3,4)+i \epsilon^{\alpha \beta} k_{1 \alpha} k_{2 \beta} \psi_{u u d g}^{(16)}(1,2,3,4)\right)\right) \\
& \times \frac{\epsilon^{j k l} T_{l i}^{a}}{2}\left[u_{i \uparrow}^{\dagger}(1)\left(u_{j \uparrow}^{\dagger}(2) d_{k_{\downarrow}}^{\dagger}(3)-d_{j \uparrow}^{\dagger}(2) u_{k \downarrow}^{\dagger}(3)\right) g_{\downarrow}^{a \dagger}(4)\right] \\
& +\left(k_{1 \perp}^{+}\left(\psi_{u u d g}^{(17)}(1,2,3,4)+i \epsilon^{\alpha \beta} k_{2 \alpha} k_{3 \beta} \psi_{u u d g}^{(18)}(1,2,3,4)\right)\right. \\
& \left.+k_{2 \perp}^{+}\left(\psi_{u u d g}^{(19)}(1,2,3,4)+i \epsilon^{\alpha \beta} k_{1 \alpha} k_{3 \beta} \psi_{u u d g}^{(20)}(1,2,3,4)\right)\right) \\
& \left.\times \frac{\epsilon^{j k l} T_{l i}^{a}}{2}\left[u_{i \downarrow}^{\dagger}(1)\left(u_{j \uparrow}^{\dagger}(2) d_{k \uparrow}^{\dagger}(3)-d_{j \uparrow}^{\dagger}(2) u_{k \uparrow}^{\dagger}(3)\right) g_{\downarrow}^{a \dagger}(4)\right]\right\}|0\rangle .
\end{aligned}
$$

The symmetry between 2 and 3 leads to $\psi_{\text {uudg }}^{(17)}(1,2,3,4)=-\psi_{\text {uudg }}^{(17)}(1,3,2,4)$ and $\psi_{\text {uudg }}^{(18)}(1,2,3,4)=\psi_{\text {uudg }}^{(18)}(1,3,2,4)$. In the second case, we define 4 independent amplitudes

$$
\begin{aligned}
|P \uparrow\rangle_{u u d g}^{l_{z}=1}= & \int d[1] d[2] d[3] d[4]\left(k_{1 \perp}^{+}\left(\psi_{u u d g}^{(21)}(1,2,3,4)+i \epsilon^{\alpha \beta} k_{2 \alpha} k_{3 \beta} \psi_{u u d g}^{(22)}(1,2,3,4)\right)\right. \\
& \left.+k_{2 \perp}^{+}\left(\psi_{u u d g}^{(23)}(1,2,3,4)+i \epsilon^{\alpha \beta} k_{1 \alpha} k_{3 \beta} \psi_{u u d g}^{(24)}(1,2,3,4)\right)\right) \\
& \times \frac{\epsilon^{j k l} T_{l i}^{a}}{2}\left[u_{i \downarrow}^{\dagger}(1)\left(u_{j \downarrow}^{\dagger}(2) d_{k \downarrow}^{\dagger}(3)-d_{j \downarrow}^{\dagger}(2) u_{k \downarrow}^{\dagger}(3)\right) g_{\uparrow}^{a \dagger}(4)\right]|0\rangle,
\end{aligned}
$$

where $\psi_{\text {uudg }}^{(21)}(1,2,3,4)=-\psi_{\text {uudg }}^{(21)}(1,3,2,4)$ and $\psi_{\text {uudg }}^{(22)}(1,2,3,4)=\psi_{\text {uudg }}^{(22)}(1,3,2,4)$.

For $l_{z}=2$, the parton helicity must be $\lambda_{u u d}=-1 / 2$ and $\lambda_{g}=-1$. We define 15 independent amplitudes

$$
\begin{aligned}
|P \uparrow\rangle_{\text {uudg }}^{l_{z}=2}= & \int d[1] d[2] d[3] d[4]\left\{\left(\left(k_{1 \perp}^{+}\right)^{2}\left(\psi_{\text {uudg }}^{(25)}(1,2,3,4)+i \epsilon^{\alpha \beta} k_{2 \alpha} k_{3 \beta} \psi_{\text {uudg }}^{(26)}(1,2,3,4)\right)\right.\right. \\
& +\left(k_{2 \perp}^{+}\right)^{2}\left(\psi_{\text {uudg }}^{(27)}(1,2,3,4)+i \epsilon^{\alpha \beta} k_{1 \alpha} k_{3 \beta} \psi_{\text {uudg }}^{(28)}(1,2,3,4)\right) \\
& +\left(k_{3 \perp}^{+}\right)^{2}\left(\psi_{\text {uudg }}^{(29)}(1,2,3,4)+i \epsilon^{\alpha \beta} k_{1 \alpha} k_{2 \beta} \psi_{\text {uudg }}^{(30)}(1,2,3,4)\right) \\
& \left.+k_{1 \perp}^{+} k_{2 \perp}^{+} \psi_{\text {uudg }}^{(31)}(1,2,3,4)+k_{1 \perp}^{+} k_{3 \perp}^{+} \psi_{\text {uudg }}^{(32)}(1,2,3,4)+k_{2 \perp}^{+} k_{3 \perp}^{+} \psi_{\text {uudg }}^{(33)}(1,2,3,4)\right) \\
& \times \frac{\epsilon^{j k l} T_{l i}^{a}}{2}\left[u_{i \downarrow}^{\dagger}(1)\left(u_{j \uparrow}^{\dagger}(2) d_{k_{\downarrow}}^{\dagger}(3)-d_{j \uparrow}^{\dagger}(2) u_{k_{\downarrow}}^{\dagger}(3)\right) g_{\downarrow}^{a \dagger}(4)\right] \\
& +\left(\left(k_{1 \perp}^{+}\right)^{2}\left(\psi_{\text {uudg }}^{(34)}(1,2,3,4)+i \epsilon^{\alpha \beta} k_{2 \alpha} k_{3 \beta} \psi_{\text {uudg }}^{(35)}(1,2,3,4)\right)\right. \\
& +\left(k_{2 \perp}^{+}\right)^{2}\left(\psi_{u u d g}^{(36)}(1,2,3,4)+i \epsilon^{\alpha \beta} k_{1 \alpha} k_{3 \beta} \psi_{u u d g}^{(37)}(1,2,3,4)\right)
\end{aligned}
$$




$$
\begin{aligned}
& \left.+k_{1 \perp}^{+} k_{2 \perp}^{+} \psi_{\text {uudg }}^{(38)}(1,2,3,4)+k_{2 \perp}^{+} k_{3 \perp}^{+} \psi_{u u d g}^{(39)}(1,2,3,4)\right) \\
& \left.\times \frac{\epsilon^{j k l} T_{l i}^{a}}{2}\left[u_{i \uparrow}^{\dagger}(1)\left(u_{j \downarrow}^{\dagger}(2) d_{k \downarrow}^{\dagger}(3)-d_{j \downarrow}^{\dagger}(2) u_{k \downarrow}^{\dagger}(3)\right) g_{\downarrow}^{a \dagger}(4)\right]\right\}|0\rangle,
\end{aligned}
$$

where $\psi_{u u d g}^{(34,39)}(1,2,3,4)=-\psi_{u u d g}^{(34,39)}(1,3,2,4)$ and $\psi_{u u d g}^{(35)}(1,2,3,4)=\psi_{u u d g}^{(35)}(1,3,2,4)$.

For $l_{z}=3$, the parton helicity must be $\lambda_{u u d}=-3 / 2$ and $\lambda_{g}=-1$. We define 8 independent amplitudes:

$$
\begin{aligned}
|P \uparrow\rangle_{u u d g}^{l_{z}=3}= & \int d[1] d[2] d[3] d[4]\left(\left(k_{1 \perp}^{+}\right)^{3}\left(\psi_{\text {uudg }}^{(40)}(1,2,3,4)+i \epsilon^{\alpha \beta} k_{2 \alpha} k_{3 \beta} \psi_{\text {uudg }}^{(41)}(1,2,3,4)\right)\right. \\
& +\left(k_{2 \perp}^{+}\right)^{3}\left(\psi_{\text {uudg }}^{(42)}(1,2,3,4)+i \epsilon^{\alpha \beta} k_{1 \alpha} k_{3 \beta} \psi_{u u d g}^{(43)}(1,2,3,4)\right) \\
& +\left(k_{1 \perp}^{+}\right)^{2} k_{2 \perp}^{+} \psi_{u u d g}^{(44)}(1,2,3,4)+\left(k_{2 \perp}^{+}\right)^{2} k_{1 \perp}^{+} \psi_{u u d g}^{(45)}(1,2,3,4) \\
& \left.+\left(k_{2 \perp}^{+}\right)^{2} k_{3 \perp}^{+} \psi_{u u d g}^{(46)}(1,2,3,4)+k_{1 \perp}^{+} k_{2 \perp}^{+} k_{3 \perp}^{+} \psi_{u u d g}^{(47)}(1,2,3,4)\right) \\
& \times \frac{\epsilon^{j k l} T_{l i}^{a}}{2}\left[u_{i \downarrow}^{\dagger}(1)\left(u_{j \downarrow}^{\dagger}(2) d_{k \downarrow}^{\dagger}(3)-d_{j \downarrow}^{\dagger}(2) u_{k \downarrow}^{\dagger}(3)\right) g_{\downarrow}^{a \dagger}(4)\right]|0\rangle
\end{aligned}
$$

where $\psi_{u u d g}^{(40,47)}(1,2,3,4)=-\psi_{u u d g}^{(40,47)}(1,3,2,4)$ and $\psi_{u u d g}^{(41)}(1,2,3,4)=\psi_{u u d g}^{(41)}(1,3,2,4)$.

For $l_{z}=-1$, the parton helicity must be $\lambda_{u u d}=1 / 2$ and $\lambda_{g}=1$. In this case, we define 10 independent amplitudes

$$
\begin{aligned}
|P \uparrow\rangle_{u u d g}^{l_{z}=-1}= & \int d[1] d[2] d[3] d[4]\left\{\left(k_{1 \perp}^{-}\left(\psi_{\text {uudg }}^{(48)}(1,2,3,4)+i \epsilon^{\alpha \beta} k_{2 \alpha} k_{3 \beta} \psi_{u u d g}^{(49)}(1,2,3,4)\right)\right.\right. \\
& +k_{2 \perp}^{-}\left(\psi_{u u d g}^{(50)}(1,2,3,4)+i \epsilon^{\alpha \beta} k_{1 \alpha} k_{3 \beta} \psi_{u u d g}^{(51)}(1,2,3,4)\right) \\
& \left.+k_{3 \perp}^{-}\left(\psi_{\text {uudg }}^{(52)}(1,2,3,4)+i \epsilon^{\alpha \beta} k_{1 \alpha} k_{2 \beta} \psi_{u u d g}^{(53)}(1,2,3,4)\right)\right) \\
& \times \frac{\epsilon^{j k l} T_{l i}^{a}}{2}\left[u_{i \uparrow}^{\dagger}(1)\left(u_{j \uparrow}^{\dagger}(2) d_{k \downarrow}^{\dagger}(3)-d_{j \uparrow}^{\dagger}(2) u_{k \downarrow}^{\dagger}(3)\right) g_{\uparrow}^{a \dagger}(4)\right] \\
& +\left(k_{1 \perp}^{-}\left(\psi_{u u d g}^{(54)}(1,2,3,4)+i \epsilon^{\alpha \beta} k_{2 \alpha} k_{3 \beta} \psi_{u u d g}^{(55)}(1,2,3,4)\right)\right. \\
& \left.+k_{2 \perp}^{-}\left(\psi_{u u d g}^{(56)}(1,2,3,4)+i \epsilon^{\alpha \beta} k_{1 \alpha} k_{3 \beta} \psi_{u u d g}^{(57)}(1,2,3,4)\right)\right) \\
& \left.\times \frac{\epsilon^{j k l} T_{l i}^{a}}{2}\left[u_{i \downarrow}^{\dagger}(1)\left(u_{j \uparrow}^{\dagger}(2) d_{k \uparrow}^{\dagger}(3)-d_{j \uparrow}^{\dagger}(2) u_{k \uparrow}^{\dagger}(3)\right) g_{\uparrow}^{a \dagger}(4)\right]\right\}|0\rangle,
\end{aligned}
$$

where $\psi_{\text {uudg }}^{(54)}(1,2,3,4)=-\psi_{\text {uudg }}^{(54)}(1,3,2,4)$ and $\psi_{u u d g}^{(55)}(1,2,3,4)=\psi_{u u d g}^{(55)}(1,3,2,4)$.

Finally, for $l_{z}=-2$, the parton helicity must be $\lambda_{u u d}=3 / 2$ and $\lambda_{g}=1$. We find 6 independent amplitudes

$$
\begin{aligned}
|P \uparrow\rangle_{u u d g}^{l_{z}=-2}= & \int d[1] d[2] d[3] d[4]\left(\left(k_{1 \perp}^{-}\right)^{2}\left(\psi_{u u d g}^{(58)}(1,2,3,4)+i \epsilon^{\alpha \beta} k_{2 \alpha} k_{3 \beta} \psi_{u u d g}^{(59)}(1,2,3,4)\right)\right. \\
& +\left(k_{2 \perp}^{-}\right)^{2}\left(\psi_{u u d g}^{(60)}(1,2,3,4)+i \epsilon^{\alpha \beta} k_{1 \alpha} k_{3 \beta} \psi_{u u d g}^{(61)}(1,2,3,4)\right) \\
& \left.+k_{1 \perp}^{-} k_{2 \perp}^{-} \psi_{u u d g}^{(62)}(1,2,3,4)+k_{2 \perp}^{-} k_{3 \perp}^{-} \psi_{u u d g}^{(63)}(1,2,3,4)\right) \\
& \times \frac{\epsilon^{j k l} T_{l i}^{a}}{2}\left[u_{i \uparrow}^{\dagger}(1)\left(u_{j \uparrow}^{\dagger}(2) d_{k \uparrow}^{\dagger}(3)-d_{j \uparrow}^{\dagger}(2) u_{k \uparrow}^{\dagger}(3)\right) g_{\uparrow}^{a \dagger}(4)\right]|0\rangle
\end{aligned}
$$


where $\psi_{u u d g}^{(58,63)}(1,2,3,4)=-\psi_{u u d g}^{(58,63)}(1,3,2,4)$ and $\psi_{u u d g}^{(59)}(1,2,3,4)=\psi_{u u d g}^{(59)}(1,3,2,4)$.

The wave-function amplitudes for the other color structure $\left(\epsilon^{i j l} T_{l k}^{a}+\epsilon^{i k l} T_{l j}^{a}\right) / 4$, can be defined similarly, except the sign changes in the symmetric properties for some amplitudes. We have in total of $63 \times 2=126$ independent amplitudes for the uudg Fock component in the proton.

Note that the above construction is not unique, where we have first considered the correct flavor structure for the three quarks, and then added all possible spin and color combinations. One can also start with a general spin structure for the three quarks, and then consider the isospin constraints. For example, for the total quark helicity $\lambda_{u u d}=3 / 2$, the general spin structure will be

$$
\phi(1,2,3) \epsilon^{i j l} T_{l k}^{a} u_{i \uparrow}^{\dagger}(1) u_{j \uparrow}^{\dagger}(2) d_{k \uparrow}^{\dagger}(3)|0\rangle
$$

with the color coupling $\epsilon^{i j l} T_{l k}^{a}$. This color structure says that indices $i$ and $j$ are antisymmetric, and the associated wave function amplitude $\phi(1,2,3)$ has $1 \leftrightarrow 2$ symmetry. The isospin constraint indicates the following relations for the three quark amplitude,

$$
\phi(1,2,3) \epsilon^{i j l} T_{l k}^{a}\left(u_{i \uparrow}^{\dagger}(1) u_{j \uparrow}^{\dagger}(2) d_{k \uparrow}^{\dagger}(3)+u_{i \uparrow}^{\dagger}(1) d_{j \uparrow}^{\dagger}(2) u_{k \uparrow}^{\dagger}(3)+d_{i \uparrow}^{\dagger}(1) u_{j \uparrow}^{\dagger}(2) u_{k \uparrow}^{\dagger}(3)\right)|0\rangle=0 .
$$

Applying the above relation to Eq. (61), and taking into account the $1 \rightarrow 2$ symmetry for $\phi(1,2,3)$, one has for the component,

$$
\phi^{\prime}(1,2,3) \epsilon^{i j l} T_{l k}^{a} u_{i \uparrow}^{\dagger}(1)\left(u_{j \uparrow}^{\dagger}(2) d_{k \uparrow}^{\dagger}(3)-d_{j \uparrow}^{\dagger}(2) u_{k \uparrow}^{\dagger}(3)\right)|0\rangle,
$$

where the isospin wave function for proton is explicit, and $\phi^{\prime}(1,2,3)=\phi^{\prime}(2,1,3)$. The similar analysis can be performed for the $\lambda_{\text {uud }}= \pm 1 / 2$ case. Working through all possibilities, we arrive at a different set of wave-function amplitudes, which are essentially equivalent to the above construction. As a check, for every orbital angular momentum projection $l_{z}$, we find the same number of the independent amplitudes.

\section{WAVE-FUNCTION AMPLITUDES FOR THE DELTA RESONANCE}

In this section, we extend the above classification of the wave-function amplitudes to the baryon decuplet, assuming again these are bound states. We consider one specific example, the delta resonance, and other baryon decuplets can be obtained by changing the flavor structure. The distribution amplitudes for the $\Delta^{++}$resonance have been studied in Refs. [4, 32]. $\Delta^{++}$has two independent helicity states, $\Lambda=3 / 2$ and $1 / 2$, and the other two helicity states $\Lambda=-3 / 2,-1 / 2$ can be obtained by using the $\hat{Y}$ transformation Eq. (8).

Here we classify only the three-quark amplitudes, and the total quark helicity is $\lambda_{\text {uu }}=$ $3 / 2,1 / 2,-1 / 2,-3 / 2$. We first consider $\Delta^{++}$with helicity $\Lambda=3 / 2$, The quark orbital angular momentum projection then has the following values $l_{z}=0,1,2,3$ respectively. According to the method in the previous sections, we find 6 independent wave-function amplitudes,

$$
\begin{aligned}
|\Delta, \Lambda=3 / 2\rangle_{\text {uuu }}^{l_{z}=0}= & \int d[1] d[2] d[3]\left(\psi_{\text {uuu }}^{(1)}(1,2,3)+i \epsilon^{\alpha \beta} k_{1 \alpha} k_{2 \beta} \psi_{\text {uuu }}^{(2)}(1,2,3)\right) \\
& \times \frac{\epsilon^{i j k}}{\sqrt{6}} u_{i \uparrow}^{\dagger}(1) u_{j \uparrow}^{\dagger}(2) u_{k \uparrow}^{\dagger}(3)|0\rangle,
\end{aligned}
$$




$$
\begin{aligned}
|\Delta, \Lambda=3 / 2\rangle_{\text {uuu }}^{l_{z}=1}= & \int d[1] d[2] d[3] k_{1 \perp}^{+} \psi_{\text {uuu }}^{(3)}(1,2,3) \\
& \times \frac{\epsilon^{i j k}}{\sqrt{6}} u_{i \uparrow}^{\dagger}(1) u_{j \uparrow}^{\dagger}(2) u_{k_{\downarrow}}^{\dagger}(3)|0\rangle, \\
|\Delta, \Lambda=3 / 2\rangle_{\text {uuu }}^{l_{z}=2}= & \int d[1] d[2] d[3]\left(k_{1 \perp}^{+} k_{2 \perp}^{+} \psi_{\text {uuu }}^{(4)}(1,2,3)+k_{2 \perp}^{+} k_{3 \perp}^{+} \psi_{u u u}^{(5)}(1,2,3)\right) \\
& \times \frac{\epsilon^{i j k}}{\sqrt{6}} u_{i \uparrow}^{\dagger}(1) u_{j \downarrow}^{\dagger}(2) u_{k \downarrow}^{\dagger}(3)|0\rangle, \\
|\Delta, \Lambda=3 / 2\rangle_{\text {uuu }}^{l_{z}=3}= & \int d[1] d[2] d[3] k_{1 \perp}^{+} k_{1 \perp}^{+} k_{2 \perp}^{+} \psi_{\text {uuu }}^{(6)}(1,2,3) \\
& \times \frac{\epsilon^{i j k}}{\sqrt{6}} u_{i \downarrow}^{\dagger}(1) u_{j \downarrow}^{\dagger}(2) u_{k \downarrow}^{\dagger}(3)|0\rangle,
\end{aligned}
$$

where we have used symmetry to reduce the number of independent amplitudes.

For the helicity $\Lambda=1 / 2$ state of the $\Delta^{++}$resonance, the classification is similar. The total quark helicity is the same as the above, but the orbital angular projection $l_{z}$ can be $l_{z}=0,-1,1,2$. As a result, the three quark Fock component for $\Delta^{++}(\Lambda=1 / 2)$ has the following 5 independent wave-function amplitudes,

$$
\begin{aligned}
|\Delta, \Lambda=1 / 2\rangle_{\text {uuu }}^{l_{z}=0}= & \int d[1] d[2] d[3]\left(\psi_{\text {uuu }}^{(1)}(1,2,3)+i \epsilon^{\alpha \beta} k_{1 \alpha} k_{2 \beta} \psi_{\text {uuu }}^{(2)}(1,2,3)\right) \\
& \times \frac{\epsilon^{i j k}}{\sqrt{6}} u_{i \uparrow}^{\dagger}(1) u_{j \uparrow}^{\dagger}(2) u_{k \downarrow}^{\dagger}(3)|0\rangle, \\
|\Delta, \Lambda=1 / 2\rangle_{\text {uuu }}^{l_{z}=1}= & \int d[1] d[2] d[3] k_{2 \perp}^{+} \psi_{\text {uuu }}^{(3)}(1,2,3) \\
& \times \frac{\epsilon^{i j k}}{\sqrt{6}} u_{i \uparrow}^{\dagger}(1) u_{j \downarrow}^{\dagger}(2) u_{k \downarrow}^{\dagger}(3)|0\rangle, \\
|\Delta, \Lambda=1 / 2\rangle_{\text {uuu }}^{l_{z}=-1}= & \int d[1] d[2] d[3] k_{2 \perp}^{-} \psi_{u u u}^{(4)}(1,2,3) \\
& \times \frac{\epsilon^{i j k}}{\sqrt{6}} u_{i \uparrow}^{\dagger}(1) u_{j \uparrow}^{\dagger}(2) u_{k \uparrow}^{\dagger}(3)|0\rangle, \\
|\Delta, \Lambda=1 / 2\rangle_{u u u}^{l_{z}=2}= & \int d[1] d[2] d[3] k_{1 \perp}^{+} k_{2 \perp}^{+} \psi_{u u u}^{(5)}(1,2,3) \\
& \times \frac{\epsilon^{i j k}}{\sqrt{6}} u_{i \downarrow}^{\dagger}(1) u_{j \downarrow}^{\dagger}(2) u_{k \downarrow}^{\dagger}(3)|0\rangle .
\end{aligned}
$$

We will not go beyond the three-quark Fock components, although those can be classified similarly.

Since the flavor structure for the baryon decuplet is completely symmetric, the light-cone expansion for the other states in the decuplet can be easily written down from the above results, apart from that the flavor structure need to be replaced accordingly. For example, the $\Delta^{+}$resonance has the symmetric flavor structure in the form of $(u u d+u d u+d u u) / \sqrt{3}$. Substituting this into the above equations, the light-cone expansion of $\Delta^{+}$for the three quark Fock component can be obtained. These amplitudes, together with those for the proton, are needed to calculate the proton-delta transition form factors in the asymptotic limit of QCD [33, 34, 35]. 


\section{ASYMPTOTIC SCALING OF WAVE-FUNCTION AMPLITUDES}

One of the important applications of the light-cone wave-function amplitudes is to calculate hard exclusive processes. The relative importance of a particular amplitude in a process can be determined from its scaling behavior when the parton transverse-momenta become large. In this section, we follow the discussion in Ref. [6] and derive a general power counting rule for the light-cone amplitudes of any Fock components of a hadron state. As examples, we consider the asymptotic scaling of some amplitudes defined above for the $\pi^{+}$and proton.

Let $\psi_{n}\left(x_{i}, k_{i}, l_{z i}\right)$ be a general amplitude describing a $n$ partons Fock component of a hadron state with orbital angular momentum projection of $l_{z}=\sum_{i} l_{z i}$. To find the asymptotic behavior of $\psi_{n}\left(x_{i}, k_{i}, l_{z i}\right)$ in the limit that all transverse momenta are uniformly large, we consider the matrix element of a corresponding quark-gluon operator between the QCD vacuum and the hadron state

$$
\left\langle 0\left|\phi_{\mu_{1}}\left(\xi_{1}\right) \ldots \phi_{\mu_{n}}\left(\xi_{n}\right)\right| P \Lambda\right\rangle
$$

where $\phi$ are parton fields such as the "good" $(+)$ components of quark fields or $F^{+\alpha}$ of gluon fields, and $\mu_{i}$ are Dirac and transverse coordinate indices when appropriate. All spacetime coordinates $\xi_{i}$ are at equal light-cone time, $\xi_{i}^{+}=0$. Fourier-transforming with respect to all the spatial coordinates $\left(\xi_{i}^{-}, \xi_{i \perp}\right)$, we find the matrix element in the momentum space, $\left\langle 0\left|\phi_{\mu_{1}}\left(k_{1}\right) \ldots \phi_{\mu_{n-1}}\left(k_{n-1}\right) \phi_{\mu_{n}}(0)\right| p \Lambda\right\rangle \equiv \psi_{\mu_{1}, \ldots, \mu_{n}}\left(k_{1}, \ldots, k_{n-1}\right)$, here we have just shown $n-1$ parton momenta because of the overall momentum conservation. The matrix element can be written as a sum of terms involving projection operator $\Gamma_{\mu_{1} \ldots \mu_{n}}^{A}\left(k_{i \perp}\right)$ multiplied by scalar amplitude $\psi_{n A}\left(x_{i}, k_{i \perp}, l_{z i}\right)$ :

$$
\begin{aligned}
\left\langle 0\left|\phi_{\mu_{1}}\left(k_{1}\right) \ldots \phi_{\mu_{n-1}}\left(k_{n-1}\right) \phi_{\mu_{n}}(0)\right| p \Lambda\right\rangle & \equiv \psi_{\mu_{1}, \ldots, \mu_{n}}\left(k_{1}, \ldots, k_{n-1}\right) \\
& =\sum_{A} \Gamma_{\mu_{1} \ldots \mu_{n}}^{A}\left(k_{i \perp}\right) \psi_{n}^{(A)}\left(x_{i}, k_{i \perp}, l_{z i}\right)
\end{aligned}
$$

where the projection operator $\Gamma^{A}$ contains Dirac matrices and is a polynomial of order $l_{z}$ in parton momenta. For example, the two quark matrix element of the pion can be written as [3],

$$
\begin{aligned}
& \left\langle 0\left|\bar{d}_{+\mu}(0) u_{+\nu}\left(x, k_{\perp}\right)\right| \pi^{+}(P)\right\rangle \\
& =\left(\gamma_{5} P\right)_{\nu \mu} \psi_{u \bar{d}}^{(1)}\left(x, k_{\perp}, l_{z}=0\right)+\left(\gamma_{5} \sigma^{-\alpha}\right)_{\nu \mu} P^{+} k_{\perp \alpha} \psi_{u \bar{d}}^{(2)}\left(x, k_{\perp}, l_{z}=1\right)
\end{aligned}
$$

where the projection operators are shown manifestly. More examples for the proton matrix elements can be found in Ref. [5].

The matrix element of our interest is, in fact, a Bethe-Salpeter amplitude projected onto the light cone. One can write down formally a Bethe-Salpeter equation which includes mixing contributions from other light-cone matrix elements. In the limit of large transverse momentum $k_{i \perp}$, the Bethe-Salpeter kernels can be calculated in perturbative QCD because of asymptotic freedom. In the lowest order, the kernels consist of a minimal number of gluon and quark exchanges linking the active partons. For the lowest Fock components of the pion wave function, one gluon exchange is needed to get a large transverse momentum for both quarks [14]. As we shall see, asymptotic behavior of the wave function amplitudes depends on just the mass dimension of the kernels. 
Schematically, we have the following equation for the light-cone amplitudes,

$$
\begin{aligned}
& \psi_{\alpha_{1}, \ldots, \alpha_{n}}\left(k_{1}, \ldots, k_{n-1}\right)=\sum_{A} \Gamma_{\alpha_{1} \ldots \alpha_{n}}^{A}\left(k_{i \perp}\right) \psi_{n}^{A}\left(x_{i}, k_{i \perp}, l_{z i}\right) \\
= & \sum_{n^{\prime}, \beta_{1}, \ldots, \beta_{n^{\prime}}} \int d^{4} q_{1} \ldots d^{4} q_{n^{\prime}-1} H_{\alpha_{1} \ldots, \alpha_{n}, \beta_{1}, \ldots, \beta_{n^{\prime}}}\left(q_{i}, k_{i}\right) \psi_{\beta_{1}, \ldots, \beta_{n^{\prime}}}\left(q_{1}, \ldots, q_{n^{\prime}-1}\right)
\end{aligned}
$$

where $H_{\alpha_{1}, \ldots, \alpha_{n}, \beta_{1}, \ldots, \beta_{n^{\prime}}}$ are the Bethe-Salpeter kernels multiplied by the parton propagators. When the parton transverse momenta are uniformly large, the kernels can be approximated by a sum of perturbative diagrams. The leading contribution to the amplitudes on the left can be obtained by iterating the above equation, assuming the amplitudes under the integration sign contain no hard components. As such, the integrations over $q_{i \perp}$ can be cut-off at a scale $\mu$ where $k_{\perp} \gg \mu \gg \Lambda_{\mathrm{QCD}}$, and the $q_{i}$ dependence in $H$ can be expanded in Taylor series. In order to produce a contribution to $\psi_{n}^{(A)}\left(x_{i}, k_{i \perp}, l_{z i}\right)$, the hard kernels must contain the projection operator $\Gamma_{\alpha_{1} \ldots \alpha_{n}}^{A}\left(k_{1}, \ldots, k_{n-1}\right)$. Hence we write

$$
\begin{aligned}
& H_{\alpha_{1} \ldots, \alpha_{n}, \beta_{1}, \ldots, \beta_{n}^{\prime}}\left(q_{i}, k_{i}\right) \\
= & \sum_{A, B} \Gamma_{\alpha_{1} \ldots \alpha_{n}}^{A}\left(k_{i \perp}\right) H_{A B}\left(x_{i}, k_{i \perp}, y_{i}\right) \Gamma_{\beta_{1} \ldots \beta_{n^{\prime}}}^{B}\left(q_{i \perp}\right),
\end{aligned}
$$

where $\Gamma_{\beta_{1} \ldots \beta_{n^{\prime}}}^{B}\left(q_{i \perp}\right)$ is again a projection operator and $H_{A B}\left(x_{i}, k_{i}, y_{i}\right)$ are scalar functions of the transverse momenta $k_{i \perp}$ invariants. Substituting the above into Eq.(75) and integrating over $q_{i}^{-}$,

$$
\begin{aligned}
& \psi_{n}^{(A)}\left(x_{i}, k_{i \perp}, l_{z i}\right) \\
= & \sum_{B, \beta_{i}} \int d y_{1} \ldots d y_{n^{\prime}-1} H_{A B}\left(x_{i}, k_{i}, y_{i}\right) \int d^{2} q_{1 \perp \ldots} d^{2} q_{\left(n^{\prime}-1\right) \perp} \Gamma_{\beta_{1} \ldots \beta_{n^{\prime}}}^{B}\left(q_{i \perp}\right) \psi_{\beta_{1}, \ldots, \beta_{n^{\prime}}}\left(y_{i}, q_{i}\right) \\
= & \sum_{B, \beta_{i}, A^{\prime}} \int d y_{1} \ldots d y_{n^{\prime}-1} H_{A B}\left(x_{i}, k_{i}, y_{i}\right) \int d^{2} q_{1 \perp \ldots} d^{2} q_{\left(n^{\prime}-1\right) \perp} \Gamma_{\beta_{1} \ldots \beta_{n^{\prime}}}^{B}\left(q_{i \perp}\right) \\
& \times \Gamma_{\beta_{1} \ldots \beta_{n^{\prime}}}^{A^{\prime}}\left(q_{i \perp}\right) \psi_{n^{\prime}}^{\left(A^{\prime}\right)}\left(y_{i}, q_{i \perp}, l_{z i}^{\prime}\right),
\end{aligned}
$$

where the integrations over $q_{i \perp}$ are non-zero only when the angular momentum content of $\Gamma^{B}$ and $\Gamma^{A^{\prime}}$ is the same. Now the large momenta $k_{i \perp}$ are entirely isolated in $H_{A B}$ which does not depend on any soft scale. The asymptotic behavior of $\psi_{n}^{(A)}\left(k_{i \perp}\right)$ is determined by the mass dimension of $H_{A B}$, which can be obtained, in principle, by working through one of the simplest perturbative diagrams.

A much simpler way to proceed is to use light-cone power counting in which the longitudinal mass dimension, such as $P^{+}$, can be ignored because of the boost invariance of the above equation along the $z$ direction. We just need to focus on the transverse dimensions. The mass dimension of the light-cone wave function amplitude $\psi_{n}$ can be determined as follows: Assume the hadron state is normalized relativistically $\left\langle P \mid P^{\prime}\right\rangle=2 E(2 \pi)^{3} \delta^{3}\left(\overrightarrow{P^{\prime}}-\vec{P}\right)$, $|P\rangle$ has mass dimension -1 . Likewise, the parton creation operator $a_{i}^{\dagger}$ has mass dimension -1 . Given these, the mass dimension of $\psi_{n}$ is $-\left(n+\left|l_{z}\right|-1\right)$ according to Eq. (17). The mass dimension of $\psi_{n(i j)}$ term in (7), however, is $-\left(n+\left|l_{z}\right|+1\right)$ which can be accounted for by the previous formula with an effective angular momentum projection $\left|l_{z}\right|+2$. 
Now considering Eq. (177), since the mass dimension of the amplitude $\psi_{n}^{(A)}\left(x_{i}, k_{i \perp}, l_{z i}\right)$ is $-\left(n+\left|l_{z}\right|-1\right)$, that of $\Gamma^{B} \Gamma^{A^{\prime}}$ is $2\left|l_{z}^{\prime}\right|$, and the integration measure $2\left(n^{\prime}-1\right)$, a balance of the mass dimension yields $\left[H_{A B}\right]=-\left(n-1+\left|l_{z}\right|\right)-\left(n^{\prime}-1+\left|l_{z}^{\prime}\right|\right)$. Therefore, the leading behavior of the wave function amplitude goes as [ 6$]$

$$
\psi_{n}^{(A)}\left(x_{i}, k_{i \perp}, l_{z i}\right) \sim \frac{1}{\left(k_{\perp}^{2}\right)^{\left[n+\left|l_{z}\right|+\min \left(n^{\prime}+\left|l_{z}^{\prime}\right|\right)\right] / 2-1}},
$$

which is determined by a mixing amplitude with smallest $n^{\prime}+\left|l_{z}^{\prime}\right|$. Since the wave function amplitude has mass dimension of $-\left(n+\left|l_{z}\right|-1\right)$, the coefficient of the asymptotic form must have a soft mass dimension $\Lambda_{\mathrm{QCD}}^{\min \left(n^{\prime}+\left|l_{z}^{\prime}\right|\right)-1}$.

We have the following selection rules for amplitude mixings. First of all, because of angular momentum conservation, wave function amplitudes belonging to different hadron helicity states do not mix. Second, because of the vector coupling in QCD, the quark helicity in a hard process does not change. Therefore, the pion amplitude $\psi_{u \bar{d}}^{(2)}$ does not mix with $\psi_{u \bar{d}}^{(1)}$ because the total quark helicity differs. An example of the nontrivial amplitude mixing is between the pion's two-quark-one-gluon and two-quark amplitudes.

The power counting rule Eq. (78) can be used to predict the scaling behaviors for all the light-cone wave function amplitudes we have written down in the above for the mesons and baryons. We will not go into much details about these predictions, rather we consider some examples for the $\pi^{+}$and proton. According to Eq. (78), the scaling behaviors for the two-parton light-cone amplitudes of $\pi^{+}$are

$$
\psi_{u \bar{d}}^{(1)}(1,2) \sim 1 / k_{\perp}^{2}, \quad \psi_{u \bar{d}}^{(2)}(1,2) \sim 1 / k_{\perp}^{4} .
$$

The $u \bar{d} g$ Fock amplitudes have the following scaling,

$$
\psi_{u \bar{d} g}^{(1,3,4,5,6)}(1,2,3) \sim 1 / k_{\perp}^{4}, \quad \psi_{u \bar{d} g}^{(2,7,8,9)}(1,2,3) \sim 1 / k_{\perp}^{6},
$$

where the mixings with the two-parton components give the dominant contribution at large $k_{\perp}$.

For the three quark Fock component of the proton, we have the following scaling behaviors for the light-cone amplitudes,

$$
\psi_{\text {uud }}^{(1)} \sim 1 / k_{\perp}^{4}, \quad \psi_{\text {uud }}^{(2,3,4,5)} \sim 1 / k_{\perp}^{6}, \quad \psi_{u u d}^{(6)} \sim 1 / k_{\perp}^{8} .
$$

Here, the scaling behaviors of $\psi_{u u d}^{(1,3,4,5,6)}$ at large $k_{\perp}$ are determined by self mixings, while that of $\psi_{u u d}^{(2)}$ is determined by mixing with $\psi_{u u d}^{(1)}$.

\section{SUMMARY AND CONCLUSION}

Following Ref. 6], we studied in this paper how to classify the independent wave-function amplitudes for a hadron state. We discussed in detail how the spin, flavor (for quark) and color of the partons are systematically coupled. We have found the these amplitudes for pion and proton up to and including four partons. We also worked out the leading light-cone wave amplitudes for the $\Delta$ resonance and the $\rho$ meson. 
A general power counting rule for the light-cone wave function amplitude has been derived based on perturbative QCD [6]. Using this rule, we have predicted the asymptotic scaling behavior of a number of amplitudes for $\pi^{+}$and the proton. This general power counting rule can be used as a constraint in modeling the light-cone wave function amplitudes.

Many applications can be made based on the formalism presented here. One example is the generalized power counting rule for high energy exclusive processes [6], including processes involving nonzero parton orbital angular momentum and hadron helicity flip. A number of processes have been briefly discussed in [6]. A more detailed discussion of the generalized counting rule will be presented elsewhere. In a different direction, one can also parameterize the light-cone wave function amplitudes and fit them to many relevant experiment data, such as the elastic form factors, parton distributions, and generalized parton distributions.

X. J. and F. Y. were supported by the U. S. Department of Energy via grant DE-FG0293ER-40762. J.P.M. was supported by National Natural Science Foundation of P.R. China through grand No.19925520.

[1] S. J. Brodsky, H.-C. Pauli, and S. S. Pinsky, Phys. Rept. 301, 299 (1998), hep-ph/9705477.

[2] M. Burkardt, Adv. Nucl. Phys. 23, 1 (1996), hep-ph/9505259.

[3] M. Burkardt, X. Ji, and F. Yuan, Phys. Lett. B545, 345 (2002), hep-ph/0205272.

[4] V. M. Braun, S. E. Derkachov, G. P. Korchemsky, and A. N. Manashov, Nucl. Phys. B553, 355 (1999), hep-ph/9902375.

[5] X. Ji, J.-P. Ma, and F. Yuan, Nucl. Phys. B652, 383 (2003), hep-ph/0210430.

[6] X. Ji, J.-P. Ma, and F. Yuan (2003), hep-ph/0301141.

[7] S. J. Brodsky and G. R. Farrar, Phys. Rev. Lett. 31, 1153 (1973).

[8] V. A. Matveev, R. M. Muradian, and A. N. Tavkhelidze, Nuovo Cim. Lett. 7, 719 (1973).

[9] P. L. Chung and F. Coester, Phys. Rev. D44, 229 (1991).

[10] F. Schlumpf, Phys. Rev. D47, 4114 (1993), hep-ph/9212250.

[11] J. Bolz and P. Kroll, Z. Phys. A356, 327 (1996), hep-ph/9603289.

[12] M. Diehl, T. Feldmann, R. Jakob, and P. Kroll, Eur. Phys. J. C8, 409 (1999), hep-ph/9811253.

[13] J. B. Kogut and D. E. Soper, Phys. Rev. D1, 2901 (1970).

[14] G. P. Lepage and S. J. Brodsky, Phys. Rev. D22, 2157 (1980).

[15] A. H. Mueller and J.-W. Qiu, Nucl. Phys. B268, 427 (1986).

[16] A. A. Slavnov and S. A. Frolov, Theor. Math. Phys. 73, 1158 (1987).

[17] N. V. Antonov, Theor. Math. Phys. 75, 605 (1988).

[18] Y. V. Kovchegov, Phys. Rev. D55, 5445 (1997), hep-ph/9701229.

[19] X. Ji and F. Yuan, Phys. Lett. B543, 66 (2002), hep-ph/0206057.

[20] A. V. Belitsky, X. Ji, and F. Yuan, hep-ph/0208038 (2002).

[21] D. Boer, P. J. Mulders, and F. Pijlman (2003), hep-ph/0303034.

[22] M. Jacob and G. C. Wick, Ann. Phys. 7, 404 (1959).

[23] G. P. Lepage and S. J. Brodsky, Phys. Lett. B87, 359 (1979).

[24] V. L. Chernyak and A. R. Zhitnitsky, Phys. Rept. 112, 173 (1984).

[25] V. M. Braun and I. E. Halperin, Z. Phys. C48, 239 (1990).

[26] S. J. Brodsky and M. Karliner, Phys. Rev. Lett. 78, 4682 (1997), hep-ph/9704379.

[27] V. L. Chernyak and I. R. Zhitnitsky, Nucl. Phys. B246, 52 (1984). 
[28] P. Ball, V. M. Braun, Y. Koike, and K. Tanaka, Nucl. Phys. B529, 323 (1998), hep$\mathrm{ph} / 9802299$.

[29] I. D. King and C. T. Sachrajda, Nucl. Phys. B279, 785 (1987).

[30] V. L. Chernyak, A. A. Ogloblin, and I. R. Zhitnitsky, Z. Phys. C42, 583 (1989).

[31] V. Braun, R. J. Fries, N. Mahnke, and E. Stein, Nucl. Phys. B589, 381 (2000), hep$\mathrm{ph} / 0007279$.

[32] G. R. Farrar, H. Zhang, A. A. Ogloblin, and I. R. Zhitnitsky, Nucl. Phys. B311, 585 (1989).

[33] S. J. Brodsky, G. P. Lepage, and S. A. A. Zaidi, Phys. Rev. D23, 1152 (1981).

[34] C. E. Carlson, Phys. Rev. D34, 2704 (1986).

[35] C. E. Carlson, M. Gari, and N. G. Stefanis, Phys. Rev. Lett. 58, 1308 (1987). 